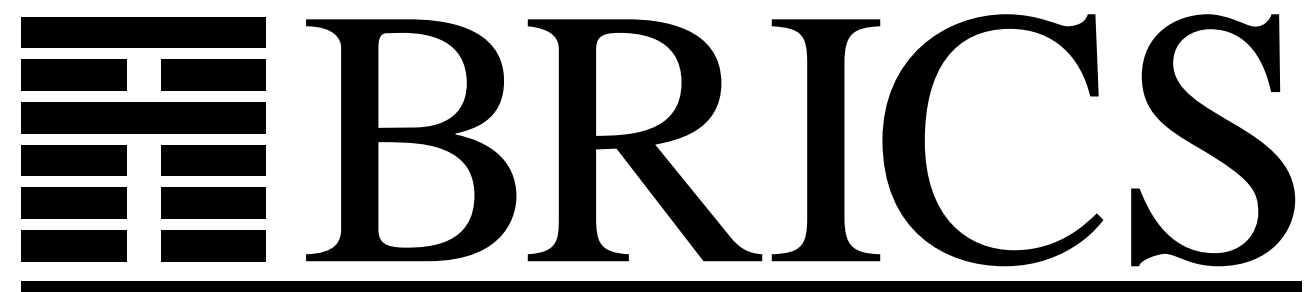

Basic Research in Computer Science

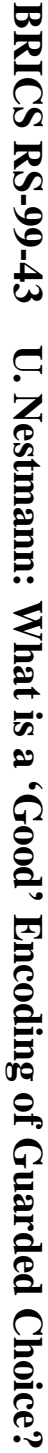

What is a

'Good' Encoding of Guarded Choice?

Uwe Nestmann

BRICS Report Series

RS-99-43 
Copyright (c) 1999, Uwe Nestmann.

BRICS, Department of Computer Science University of Aarhus. All rights reserved.

Reproduction of all or part of this work is permitted for educational or research use on condition that this copyright notice is included in any copy.

See back inner page for a list of recent BRICS Report Series publications. Copies may be obtained by contacting:

\author{
BRICS \\ Department of Computer Science \\ University of Aarhus \\ Ny Munkegade, building 540 \\ DK-8000 Aarhus C \\ Denmark \\ Telephone: +4589423360 \\ Telefax: $\quad+4589423255$ \\ Internet: BRICS@brics.dk
}

BRICS publications are in general accessible through the World Wide Web and anonymous FTP through these URLs:

http://www.brics.dk

ftp: / / ftp.brics.dk

This document in subdirectory RS/99/43/ 


\title{
What is a 'Good' Encoding of Guarded Choice?*
}

\author{
Uwe Nestmann $^{\dagger}$ \\ BRICS ${ }^{\ddagger}$, Aalborg University, Denmark
}

\begin{abstract}
The $\pi$-calculus with synchronous output and mixed-guarded choices is strictly more expressive than the $\pi$-calculus with asynchronous output and no choice. This result was recently proved by Palamidessi and, as a corollary, she showed that there is no fully compositional encoding from the former into the latter that preserves divergencefreedom and symmetries. This paper argues that there are nevertheless 'good' encodings between these calculi.

In detail, we present a series of encodings for languages with (1) input-guarded choice, (2) both input- and output-guarded choice, and (3) mixed-guarded choice, and investigate them with respect to compositionality and divergence-freedom. The first and second encoding satisfy all of the above criteria, but various 'good' candidates for the third encoding - inspired by an existing distributed implementation-invalidate one or the other criterion. While essentially confirming Palamidessi's result, our study suggests that the combination of strong compositionality and divergence-freedom is too strong for more practical purposes.
\end{abstract}

*This is a revised and slightly extended version of a paper published in the Proceedings of EXPRESS'97 (4th International Workshop on Expressiveness in Concurrency), volume 7 of Electronic Notes in Theoretical Computer Science, Elsevier Science Publishers.

${ }^{\dagger}$ The work was mainly carried out while the author was supported by a post-doc fellowship from ERCIM (European Research Consortium for Informatics and Mathematics) and partially supported by the ESPRIT CONFER-2 WG-21836. The work was revised and completed under a grant from BRICS.

$\ddagger$ Basic Research in Computer Science, Centre of the Danish National Research Foundation. 


\section{Contents}

1 Introduction 1

2 Technical Preliminaries $\quad 5$

3 Implementing Separate Choice $\quad 7$

3.1 Input-guarded choice . . . . . . . . . . . . . . . . . . . . 7

3.2 Output-guarded choice . . . . . . . . . . . . . . . . 8

4 Implementing Mixed Choice $\quad 10$

4.1 A randomized solution . . . . . . . . . . . . . . . . . 11

4.2 A 'bakery' algorithm . . . . . . . . . . . . . . . . . . . . 13

4.3 A 'practical' solution . . . . . . . . . . . . . . . . . 15

$\begin{array}{lll}5 & \text { Full abstraction } & 17\end{array}$

5.1 Gradual commitments . . . . . . . . . . . . . . . . . . . . 17

5.2 On barbed bisimulation . . . . . . . . . . . . . . . . . . 18

5.3 Adequacy via restriction . . . . . . . . . . . . . . . . 23

6 Conclusion $\quad 26$

$\begin{array}{ll}\text { A Channel managers are not uniform } & 27\end{array}$

B Type-checking partial deadlock-freedom 28

B.1 Types for reliable channels . . . . . . . . . . . . . . . . . . 28

B.2 Separate choice . . . . . . . . . . . . . . . . . . . . 30

B.3 Mixed choice . . . . . . . . . . . . . . . . . . . . . . . 32 


\section{Introduction}

The invention of the $\pi$-calculus [MPW92] by Milner, Parrow, and Walker, has triggered a wide range of encodings of other calculi into it, due to its well-developed semantic theory, but also because of the similarities between encodings and actual implementations by the use of namepassing. Soon the question arose, which operators would be responsible for this surprisingly expressive power of the original $\pi$-calculus. This paper contributes to the understanding of the role of choice operators for the expressiveness of the $\pi$-calculus.

A widely-used method for measuring the relative expressiveness of calculi is by (mutual) encodings. A calculus is considered more expressive than another, if it represents the target language of an encoding of the other calculus. The meaningfulness of such propositions rests on the (syntactic and semantic) properties that are preserved and/or reflected by the encoding. An example criterion for being a 'good' encoding is the popular notion of full abstraction: in the context of process calculi, it requires that the equivalence of terms is both preserved and reflected (cf. Sangiorgi [San93]). Of course, the choice of equivalence is crucial. Weak bisimulation equivalences and congruences have become prominent in this area, because they permit abstraction from internal steps that might be added by an encoding, and also because they provide handy proof techniques. Yet, weak bisimulation is not the only interesting equivalence relation; in particular, it is insensitive to divergence. Consequently, an encoding that introduces infinite loops may nevertheless be fully abstract with respect to weak bisimulation; for stating that an encoding is divergence-free, we need additional arguments (or a different equivalence). If full abstraction can not be achieved for any known equivalences, then the mere preservation of states' properties like

- deadlock-freedom: it is possible to perform some transition

- livelock-freedom: it is always possible to escape infinite internal computations

- divergence-freedom: there are no infinite internal computations

may also be used to argue that an encoding can be accepted as 'good'.

More traditional methods of measuring the expressiveness of models for concurrency are by checking the existence of solutions for certain well-known problems, e.g. algorithms for mutual exclusion [RL94], consensus [Ben83], and leader election [Bou88] in symmetric distributed systems, or else by checking their Turing power via the construction of random access machines [BGZ97]. Here, a model (possibly provided by a process calculus) is considered more expressive than another, if it provides solutions to more problems.

Many variations of the above-mentioned measures have been applied to study the expressiveness of a whole family of name-passing process calculi. Calculi with asynchronous name-passing like the $\nu$-calculus [HT92] and the corresponding variant of the choice-free $\pi$ calculus [Bou92] have recently attracted particular interest, since they still have surprisingly expressive power. To study their expressiveness relative to the original $\pi$-calculus [MPW92], the existence of 'good' encodings of operators for synchronous output and guarded choice 


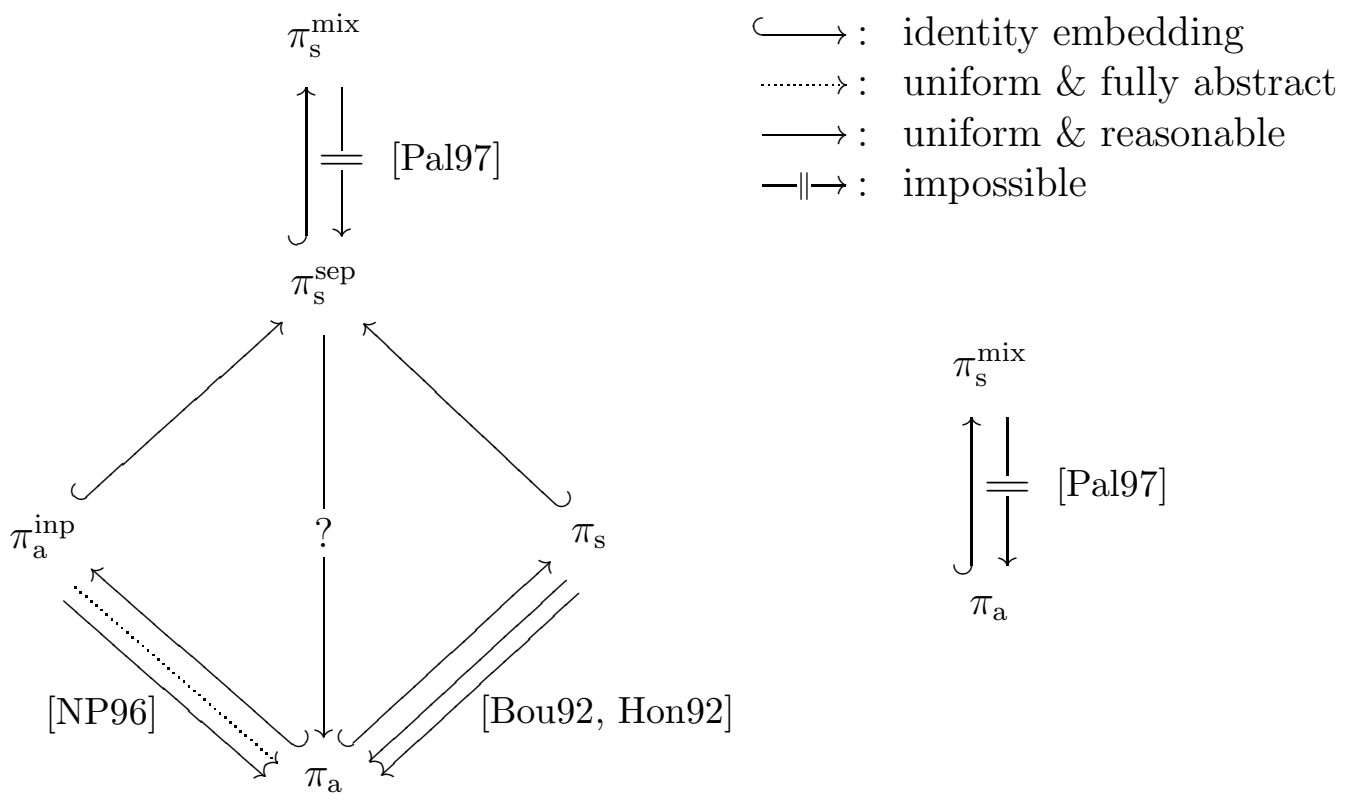

Figure 1: Encodings for choice and synchrony in the asynchronous $\pi$-calculus

(we are not concerned with matching operators) is investigated. Figure 1 summarizes the respective results that are known from the literature, on which we comment in the following paragraphs. The subscripts $a$ and $s$ denote calculi with asynchronous and synchronous output, respectively, whereas the superscripts inp, sep, and mix denote, which kind of guarded choice is contained in the language: input-guarded, separate (choices with either only inputor only output-guards), or mixed.

Synchronous output can be encoded by means of asynchronous output using explicit acknowledgement channels: Boudol [Bou92] provided an encoding from $\pi_{\mathrm{s}}$ into $\pi_{\mathrm{a}}$ and proved its correctness as adequacy (just the reflection part of full abstraction) with respect to Morrisstyle contextual congruence; Honda [Hon92] gave a more efficient (in terms of number of low-level steps needed for implementing one high-level step) encoding and showed correctness as adequacy with respect to some weak bisimulation, and as preservation of satisfaction for logical formulae via an embedding of a modal logic.

Choice operators play a crucial role in assessing the expressiveness of the original (synchronous) $\pi$-calculus and its asynchronous descendants, since they are usually present in the former, but not [HT92, Bou92] (or only restricted [ACS98]) in the latter. Nestmann and Pierce showed in [NP96] that at least input-guarded choice can be encoded into $\pi_{\mathrm{a}}$ and proven to be fully abstract with respect to weak bisimulation [HT92, ACS98] for an encoding with infinite loops, and fully abstract with respect to coupled simulation for a divergence-free encoding. 
However, Palamidessi proved that there is no uniform encoding from $\pi_{\mathrm{s}}^{\text {mix }}$ into $\pi_{\mathrm{a}}$ that preserves a reasonable semantics. In other words, it is impossible to encode mixed-guarded choice with only asynchronous name-passing, when imposing Palamidessi's criteria:

uniform means, according to Palamidessi [Pal97]: for all source terms $P, P_{1}$, and $P_{2}$,

$$
\begin{aligned}
\llbracket \sigma(P) \rrbracket & =\sigma(\llbracket P \rrbracket) \\
\llbracket P_{1} \mid P_{2} \rrbracket & =\llbracket P_{1} \rrbracket \mid \llbracket P_{2} \rrbracket
\end{aligned}
$$

where $\sigma$ denotes an injective renaming function. While the first condition merely requires that the candidate encoding be compatible with the renaming of free channels, the second condition represents the requirement that an encoding of mixed-guarded choice should be 'truly distributed', in the sense that it is not allowed to have a mediating process $M$, as in

$$
\llbracket P_{1} \mid P_{2} \rrbracket=\left(\boldsymbol{\nu} x_{1}, \ldots, x_{n}\right)\left(\llbracket P_{1} \rrbracket|M| \llbracket P_{2} \rrbracket\right)
$$

which could monitor parallel activities via the internal names $x_{1}, \ldots, x_{n}$.

reasonable means, according to Palamidessi [Pal97]: "We call reasonable a semantics which distinguishes two processes $P$ and $Q$ whenever in some computation of $P$ the actions on certain intended channels are different from those in any computation of $Q$." This includes sensitivity to divergence since an action on an intended channel in some computation of $P$ is required to happen in any computation of $Q$, so infinite loops in computations of $Q$ that do not mention the intended action are detected.

Palamidessi's impossibility theorem for encodings of mixed choice is a corollary of her formal separation result between $\pi_{\mathrm{s}}^{\mathrm{mix}}$ and $\pi_{\mathrm{a}}$ (and also $\pi_{\mathrm{s}}^{\mathrm{sep}}$ ). Similar to previous work of Bougé [Bou88] within the setting of CSP, it is based on the ability or inability of the calculi to express leader election algorithms in symmetric networks (here: of $\pi$-calculus processes). Such algorithms require the ability to break symmetries in communication graphs, like the atomic agreement of two processes about two values (e.g. the process_id of the leader). $\pi_{\mathrm{s}}^{\text {mix }}$ can break such symmetries, e.g. in the parallel composition of 'symmetric' choices

$$
P\left|Q \stackrel{\text { def }}{=} y_{0} ![0] . P_{0}+y_{1} ?[x] . P_{1}\right| y_{0} ?[x] \cdot Q_{0}+y_{1} ![1] \cdot Q_{1}
$$

where symmetry means that the program code of $P$ and $Q$ is identical under structural congruence and renaming of process_id's modulo 2, we end up with either of the asymmetric systems $P_{0} \mid Q_{0}\{\% / x\}$ or $P_{1}\{1 / x\} \mid Q_{1}$. In contrast, the above symmetric system could not be written in $\pi_{\mathrm{a}}$ since mixed-guarded choice is not a part of this language. Instead, corresponding systems with concurrently enabled input- and output-actions (see the diagram aside for a process which mimics the behavior of the above $P$ ) would behave under the regime of a confluence property. Here, since both $P$ and the corresponding $Q$ would behave confluently, the symmetry of $P \mid Q$ would be preserved under computation, i.e. no leader could be elected (an attempt for leader

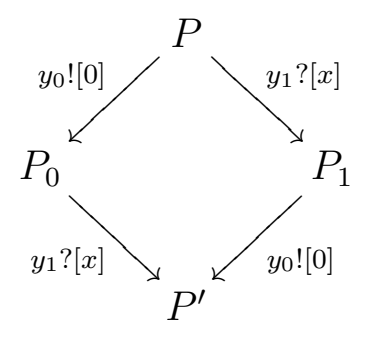


election in $\pi_{\mathrm{a}}$ would go on for an infinite amount of time, while leader election in $\pi_{\mathrm{s}}$ succeeds in finite time). Since encodings that satisfy Palamidessi's uniformity requirements preserve symmetry of networks, and since 'reasonable' semantics are divergence-sensitive, the impossibility result can be derived.

There has been remarkable interest in concurrent programming languages that include mixed choice for channel-based synchronous communication, as exemplified with Concurrent ML [Rep91] and Facile [TLK96]. Despite Palamidessi's impossibility result, there also exist algorithms for the distributed implementation of such languages, and in particular of mixed choice, e.g. by Bernstein [Ber80], Buckley and Silberschatz [BS83] and Knabe [Kna93], which all have been proven - although rather informally - to be correct or, at least, to be deadlockfree. ${ }^{1}$ The question arises how these practically satisfactory implementations relate to Palamidessi's impossibility result. It is worthwhile to ask, whether the criteria of uniformity and reasonableness are adequate or, maybe, too strong in that the above implementations invalidate them. ${ }^{2}$

This paper sheds more light on the correctness of distributed implementations of choice by formally studying choice encodings (apart from uniformity) with respect to the preservation of deadlock-, divergence-, and livelock-freedom. These properties are tightly related to Palamidessi's criteria, but they seem more precise than requiring to 'preserve a reasonable semantics'.

\section{Overview of the paper}

First, we introduce the formal $\pi$-calculus framework for our study $(\S 2)$. Then, quickly recalling the uniform encoding of input-guarded choice of [NP96], we extend it to a uniform encoding of output-guards in the context of separate choices $(\S 3)$. For this case, we show how to prove important 'reasonable' properties like deadlock- and divergence-freedom. By the attempt to smoothly reuse this encoding for the case of choices with mixed guards ( 44 ), we expose inherent deadlock-problems due to cyclic waiting and 'incestuous' self-communication. To overcome these problems, we propose various solutions, which, however, invalidate either uniformity or reasonableness. One successful possibility for an encoding of mixed choice is finally suggested by restricting the source and, at the same time, extending the target language. We also show how full abstraction results for choice encodings can be achieved with respect to barbed and other bisimulations $(\S 5)$. Finally $(\S 6)$, we offer some possible interpretations of our work.

\footnotetext{
${ }^{1}$ It has only recently (14 years after publication) turned out that the algorithm presented by Buckley and Silberschatz is not deadlock-free [KS97], although otherwise stated [BS83]. This emphasizes the need for more formal analysis of distributed implementations and, in particular, of guarded choice.

${ }^{2}$ Note also that all of the previous encodings in Figure 1 satisfy Palamidessi's criteria, with one exception: the dotted arrow from $\pi_{\mathrm{a}}^{\mathrm{inp}}$ to $\pi_{\mathrm{a}}$ indicates that one of the encodings studied in [NP96] is uniform and fully abstract, but not reasonable; this is due to infinite loops that were necessary to achieve full abstraction with respect to weak bisimulation, otherwise full abstraction could only be proved with respect to the weaker notion of coupled simulation.
} 
Throughout the paper, we emphasize the exposition of encodings, algorithms, and tradeoffs instead of just presenting the formal proofs, which are rather straightforward in most cases. Some technicalities of proofs of deadlock-freedom by using type systems, where we apply interesting more recent techniques, are assembled in the Appendix.

\section{Technical Preliminaries}

We introduce various polyadic $\pi$-calculi [Mil93] as source $(\mathbb{S})$ and target $(\mathbb{T})$ languages. Let $\mathbf{N}$ be a countable set of names, and let $\tilde{x}$ denote a finite tuple $x_{1}, \cdots, x_{n}$ of names in:

$$
\begin{aligned}
& \pi \quad:=y ![\tilde{z}] \quad \mid y ?[\tilde{x}] \\
& \mathbb{S}^{\text {mix }}: P \quad:=P|P|(\boldsymbol{\nu} y) P \quad\left|\quad y ?^{*}[\tilde{x}] . P \quad\right| \sum_{i \in I} \pi_{i} \cdot P_{i}
\end{aligned}
$$

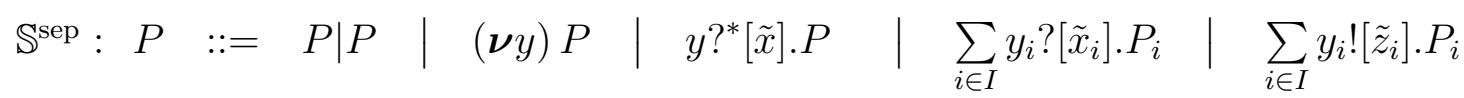

$$
\begin{aligned}
& \mathbb{S}^{\text {inp }}: P \quad:=P|P|(\boldsymbol{\nu} y) P \quad\left|\quad y ?^{*}[\tilde{x}] . P \quad\right| \quad \sum_{i \in I} y_{i} ?\left[\tilde{x}_{i}\right] . P_{i} \\
& \mathbb{T}: \quad P \quad:=\quad P|P|(\boldsymbol{\nu} y) P \quad\left|\quad y ?^{*}[\tilde{x}] . P \quad\right| \quad y ?[\tilde{x}] . P \quad \mid \quad y ![\tilde{z}]
\end{aligned}
$$

where $x, y, z \in \mathbf{N}$, and $I$ ranges over finite sets of indices $i$. The source languages $\mathbb{S}^{\Sigma}$ with $\Sigma \in\{$ mix, sep, inp $\}$ are polyadic versions of the calculi $\pi_{\mathrm{s}}^{\text {mix }}, \pi_{\mathrm{s}}^{\text {sep }}$, and $\pi_{\mathrm{a}}^{\text {inp }}$, respectively, of Figure 1. The target language $\mathbb{T}$ is defined with just asynchronous output, i.e. messages, and only single input-prefixes instead of choice and, thus, is a polyadic version of $\pi_{\mathrm{a}}$.

The informal semantics of parallel composition and restriction is as usual. In choices, we use an output guard $y ![\tilde{z}] . P$ to denote the emission of names $\tilde{z}$ along channel $y$ before behaving as $P$, and an input guard $y$ ? $[\tilde{x}] . P$ to denote the reception of arbitrary names $\tilde{z}$ along channel $y$ and afterwards behaving as $P\{\tilde{z} / \tilde{x}\}$, which denotes the simultaneous substitution of all free occurrences of names $\tilde{x}$ by the received names $\tilde{z}$, while silently performing $\alpha$-conversion, wherever necessary. A replicated input guard $y ?^{*}[\tilde{x}] . P$ denotes a process that allows us to spawn off arbitrary instances of the form $P\{\tilde{z} / \tilde{x}\}$ in parallel by repeatedly receiving names $\tilde{z}$ along channel $y$. We use $N_{1}+N_{2}$ to abbreviate binary choice (commutative and associative), and $\mathbf{0}$ to denote empty choice in $\mathbb{S}^{\Sigma}$ and the term $(\boldsymbol{\nu} x)(x ![])$ in $\mathbb{T}$.

Operator precedence is, in decreasing order of binding strength: (1) substitution, (2) prefixing, restriction, replication, (3) choice, and (4) parallel composition. A term is guarded when it occurs as a subterm of some guard. In $y ![\tilde{z}]$ and $y$ ? $[\tilde{x}], y$ is called subject, while $\tilde{x}$ and $\tilde{z}$ are called objects. The sets $f n(P)$ and $b n(P)$ of free and bound names of a process $P$, and their union $n(P)$, are defined as usual. Created names are assumed to be fresh, i.e. not occurring in any other term. 


$$
\begin{aligned}
& \mathbb{S}^{\text {mix }}, \mathbb{S}^{\text {sep }} \text { : } \\
& (\cdots+y ?[\tilde{x}] . P)|(y ![\tilde{z}] . Q+\cdots) \rightarrow P\{\tilde{z} / \tilde{x}\}| Q \\
& \mathbb{S}^{\operatorname{mix}}, \mathbb{S}^{\text {sep }} \text { : } \\
& y ?^{*}[\tilde{x}] . P|(y ![\tilde{z}] \cdot Q+\cdots) \rightarrow P\{\tilde{z} / \tilde{x}\}| Q \mid y ?^{*}[\tilde{x}] . P \\
& \mathbb{S}^{\text {inp : }} \\
& \mathbb{S}^{\text {inp }, \mathbb{T}:} \\
& \mathbb{T}: \\
& \mathbb{T}: \\
& \mathbb{T}: \\
& \text { if } \quad P \rightarrow P^{\prime} \quad \text { then } \\
& \text { if } P \rightarrow P^{\prime} \quad \text { then } \\
& \text { if } P \equiv Q \rightarrow Q^{\prime} \equiv P^{\prime} \text { then } \\
& P|Q \equiv Q| P \\
& (\cdots+y ?[\tilde{x}] \cdot P) \mid y ![\tilde{z}] \rightarrow P\{\tilde{z} / \tilde{x}\} \\
& y ?^{*}[\tilde{x}] \cdot P|y ![\tilde{z}] \rightarrow P\{\tilde{z} \tilde{x}\}| y ?^{*}[\tilde{x}] . P \\
& y ?[\tilde{x}] \cdot P \mid y ![\tilde{z}] \rightarrow P\{\tilde{z} / \tilde{x}\} \\
& \text { test } y \text { then } P \text { else } Q \mid y ![\mathrm{t}] \rightarrow P \\
& \text { test } y \text { then } P \text { else } Q \mid y ![\mathrm{f}] \rightarrow Q \\
& P|(Q \mid R) \equiv(P \mid Q)| R \\
& (\boldsymbol{\nu} x) P \rightarrow(\boldsymbol{\nu} x) P^{\prime} \\
& Q|P \rightarrow Q| P^{\prime} \\
& P \rightarrow P^{\prime} \\
& (\boldsymbol{\nu} y)(\boldsymbol{\nu} x) P \equiv(\boldsymbol{\nu} x)(\boldsymbol{\nu} y) P \\
& \text { (עy) } P \mid Q \equiv(\boldsymbol{\nu} y)(P \mid Q) \quad \text { if } \quad y \notin f n(Q)
\end{aligned}
$$

Figure 2: Reduction relation \& structural congruence

For the sake of readability in $\mathbb{T}$, we use primitive boolean names $t, f \in \mathbb{B}$ and conditional operators test $y$ then $P$ else $Q$ for destructively reading and testing the current (boolean) value on channel $y$. The above conditional is an abbreviation of $y$ ? $[x]$. if $x$ then $P$ else $Q$ with the usual meaning of if, which is only defined, if the name received for $x$ is a boolean. For $\mathbb{T}$ with booleans, we require for the grammar above that $y \in \mathbf{N}$, while $x, z \in \mathbf{V}:=\mathbf{N} \cup \mathbb{B}$. Note that booleans can be cleanly encoded into the intended target language $\mathbb{T}$ (cf. [Nes96]).

As Milner [Mil93], we assume that all processes are well-typed according to the correct use of polyadic channels, i.e. matching senders and receivers always have the same expectation about the arity or the boolean type of transmitted values. This also prevents us from restriction on, communications on, and substitution for booleans, e.g. by using structural types $T::=\mathbb{B} \mid[\tilde{T}]$ without recursion, together with straightforward typing rules.

The formal semantics for the languages $\mathbb{S}^{\Sigma}$ and $\mathbb{T}$ is presented in Figure 2 as a reduction relation $\rightarrow$ (with reflexive-transitive closure $\Rightarrow$ ) on structural congruence classes (silently including $\alpha$-conversion). The only difference among the languages is in the rules for communication, which arise from the different kinds of choices and receptors. 


$$
\begin{array}{lll}
\llbracket P_{1} \mid P_{2} \rrbracket & \stackrel{\text { def }}{=} & \llbracket P_{1} \rrbracket \mid \llbracket P_{2} \rrbracket \\
\llbracket(\boldsymbol{\nu} x) P \rrbracket & \stackrel{\text { def }}{=} & (\boldsymbol{\nu} x) \llbracket P \rrbracket \\
\llbracket \sum_{i \in I} \pi_{i} . P_{i} \rrbracket & \stackrel{\text { def }}{=} & (\boldsymbol{\nu} l)\left(l ![\mathrm{t}] \mid \prod_{i \in I} \llbracket \pi_{i} . P_{i} \rrbracket_{l}\right)
\end{array}
$$

Figure 3: Encoding scheme $\mathbb{S}^{\Sigma} \rightarrow \mathbb{T}$

\section{Implementing Separate Choice}

Intuitively, branches in a guarded choice may be seen as individual, but concurrently available processes that have to synchronize each others progress by mutual exclusion. Reminiscent of distributed implementations, we should use parallel composition to express this concurrent activity of branches.

The encoding scheme in Figure 3 implements choice-states as boolean messages on private channels $l$, so-called locks: t means that no branch in the current choice has yet been chosen, $f$ means the contrary (so the initial value must be $t$ ). Whenever (an encoding of) a branch wants to proceed, it must test its associated lock; it must also explicitly reset the lock after having tested it in order to enable competing branches to also test the choices' state. We use the scheme for several encodings. Instead of presenting them all at once, and studying their properties afterwards, we proceed stepwise, which allows us to emphasize their differences. In all cases, uniformity [Pal97] is guaranteed by the compositional encoding of parallel composition and restriction (see Appendix A).

\subsection{Input-guarded choice}

According to [NP96], input-guarded choice can be encoded as shown in Figure 3 and 4 . The only non-trivial case is for input-guards: after receiving a value from the environment, the name $l$ is used to test whether the current guard is allowed to proceed (by reading t from $l$ ), or whether it has to be aborted (by reading $\mathrm{f}$ from $l$ ) and obliged to resend the received value.

$$
\begin{array}{lll}
\llbracket y ![\tilde{z}] \rrbracket & \stackrel{\text { def }}{=} y ![\tilde{z}] \\
\llbracket y ?[\tilde{x}] . P \rrbracket l & \stackrel{\text { def }}{=} & y ?[\tilde{x}] \text {. test } l \text { then }(l ![\mathrm{f}] \mid \llbracket P \rrbracket) \text { else }(l ![\mathrm{f}] \mid y ![\tilde{x}]) \\
\llbracket y ?^{*}[\tilde{x}] . P \rrbracket & \stackrel{\text { def }}{=} & y ?^{*}[\tilde{x}] . \llbracket P \rrbracket
\end{array}
$$

Figure $4: \operatorname{sinp} \rightarrow \mathbb{T}$ 
The encoding obeys strong invariant properties on the use of locks:

- "On each lock, at most one message may ever be available at any time". This guarantee implements locking, which enables mutual exclusion.

- "Each reader of a lock eventually writes back to the lock".

This obligation enables the correct abortion of non-chosen branches.

It is crucial for the correctness that send-requests that do not lead to communication-because of the receiver being aborted - are resent, i.e. possibly passed on to another receiver waiting on the same channel. Furthermore, abortion would not be handled correctly, were we not guaranteed that, once read, lock $l$ eventually becomes available again with message $\mathrm{f}$. This encoding preserves a 'reasonable' semantics since it is fully abstract with respect to coupled simulation, which implies deadlock-freedom, and it is also divergence-free. In fact, a correctness result stronger than full abstraction holds: terms and their translations are congruent, so they cannot be distinguished by any context.

\subsection{Output-guarded choice}

If output is blocking, i.e. guarding some behavior that is only enabled if the output was successful, then synchronization is no longer local to the receiver's choice. The idea is (cf. Figure 5) that, in the target language, a sender asynchronously transmits its values $\tilde{z}$ together with a private acknowledgement channel $a$, which can be used just once by some matching receiver to signal either success or failure, i.e. either enabling the sender's continuation to proceed, or to abort it. Since output-guards are also branches in a choice whose state must be tested, the corresponding lock $r$ is, in addition to $\tilde{z}$ and $a$, transmitted to some matching receiver that then performs the required choice-test.

Input-guards, revisited The encoding is more elaborate due to the increased information that is transmitted by send-requests. Firstly, there are now two locks that have to be tested in some order. In Figure 5, we chose to test the local lock $l$ first, and only in the case of a positive outcome to test the remote lock $r$. (This particular order is useful in an actual distributed implementation, where remote communication is usually much more expensive than local communication.) Secondly, we have to use the acknowledgement channel correctly, which means that a positive acknowledgement may only be sent if both locks were tested positively. Thirdly, in the case that the test of sender's choice-lock was negative, we must not resend the send-request-instead, and only if the test of the receiver's choice-lock was positive, we have to restart the receiver process from the beginning by allowing it to try other send-requests. In Figure 5, this is implemented by recursively sending a trigger-signal to a replicated input process on $b$ that represents the receiver-loop's entry point. In order to match this protocol of synchronous outputs, the encoding of input-guarded replication has to check the sender's lock, and based on its value either to commit and trigger a copy of its continuation, or to abort the sender. 


$$
\begin{aligned}
& \llbracket y ![\tilde{z}] . P \rrbracket_{r} \quad \stackrel{\text { def }}{=}(\boldsymbol{\nu} a)(y ![r, a, \tilde{z}] \mid \text { test } a \text { then } \llbracket P \rrbracket \text { else } \mathbf{0}) \\
& \llbracket y ?[\tilde{x}] . P \rrbracket_{l} \stackrel{\text { def }}{=}(\boldsymbol{\nu} b)\left(b ![] \mid b ?^{*}[]\right. \text {. } \\
& y ?[r, a, \tilde{x}] . \\
& \text { test } l \\
& \text { then test } r \\
& \text { then } l ![\mathrm{f}]|r ![\mathrm{f}]| a ![\mathrm{t}] \mid \llbracket P \rrbracket \\
& \text { else } l ![\mathrm{t}]|r ![\mathrm{f}]| a ![\mathrm{f}] \mid b ![] \\
& \text { else } l ![\mathrm{f}] \mid y ![r, a, \tilde{x}]) \\
& \llbracket y ?^{*}[\tilde{x}] . P \rrbracket \stackrel{\text { def }}{=} y ?^{*}[r, a, \tilde{x}] \text {.test } r \text { then } r ![\mathrm{f}]|a ![\mathrm{t}]| \llbracket P \rrbracket \text { else } r ![\mathrm{f}] \mid a ![\mathrm{f}]
\end{aligned}
$$

Figure 5: $\mathbb{S}^{\text {sep }} \rightarrow \mathbb{T}$

Evaluation An encoding is deadlock/divergence-free, if it does not add deadlocks/loops to the behavior of terms: a deadlock/loop that occurs in (some derivative of) an encoded term necessarily results from a deadlock/loop already occurring in (some derivative of) the original term. Note that divergence-freedom implies livelock-freedom.

To prove deadlock-freedom, we take advantage of type information for the channels that are added in the encoding. We refine channel types according to Kobayashi's classification [Kob97], which distinguishes between reliable and unreliable channels. The following three types of channels are reliable:

- linear channels, which are used just once (like our acknowledgement channels $a$ ),

- replicated input channels, whose input ends must not occur more than once, but whose output ends may be used arbitrarily often (like our restart channels $b$ ), and

- mutex channels, which need to obey the invariants (of our lock channels $l$ ) that we mentioned on page 8; also, a message must be available right after their creation.

Kobayashi also developed a typing system that provides a behavioral property for well-typed processes: every (immediate) deadlock can only be caused by unreliable channels. A subject reduction theorem extends the proposition to deadlocks that may ever occur in derivatives of well-typed processes.

As indicated above, every channel that is added by our choice encodings, is reliable. Since we can further show that every encoded term is well-typed with respect to Kobayashi's type system (when regarding every source-level channel as unreliable), we already get the desired proposition: 
Proposition 3.2.1. $\mathbb{S}^{\mathrm{sep}} \rightarrow \mathbb{T}$ is deadlock-free.

Proof. By type-checking. More details can be found in Appendix B.

Proposition 3.2.2. $\mathbb{S}^{\mathrm{sep}} \rightarrow \mathbb{T}$ is divergence-free.

Proof. The only possibility for an encoding to add an infinite loop would be in the translation of input-guards since it is only there that we use replication. In order to trigger a copy of this replication, three conditions must be met: (1) the receiver's lock must still contain $t,(2)$ a matching send-request must be consumed from the environment, and (3) this sender's lock must contain $\mathrm{f}$. However, in this situation, by 'looping back' the consumed message will not be given back to the system - in other words, the system's state is decreased. This cannot be done infinitely often unless an infinite number of matching send-requests is produced. This, in turn, is only possible by using replication, e.g. by $(\boldsymbol{\nu} x)\left(x ![] \mid x ?^{*}[] .(x ![] \mid \cdots y ![\tilde{z}] \cdots)\right)$, but then, due to the encoding of replicated input, this replication of messages must have been already present in the source language. ${ }^{3}$

\section{Implementing Mixed Choice}

The naïve attempt is to simply reuse the encoding for separate choices of the previous section as is for encoding mixed choices. This seems sensible at first, because in both cases all inputand output-guards are branches in choices, so they should behave similarly. However, we are faced with two inherent sources of potential deadlock in the mixed setting: one is for the symmetric term $P\left|Q:=y_{0} ![0] . P_{0}+y_{1} ?[x] . P_{1}\right| y_{0} ?[x] . Q_{0}+y_{1} ![1] . Q_{1}$ of equation 4 in the Introduction, the other is for $I:=y ![z] . P+y ?[x] . Q$. The deadlock situations may become clear from a spatial representation:
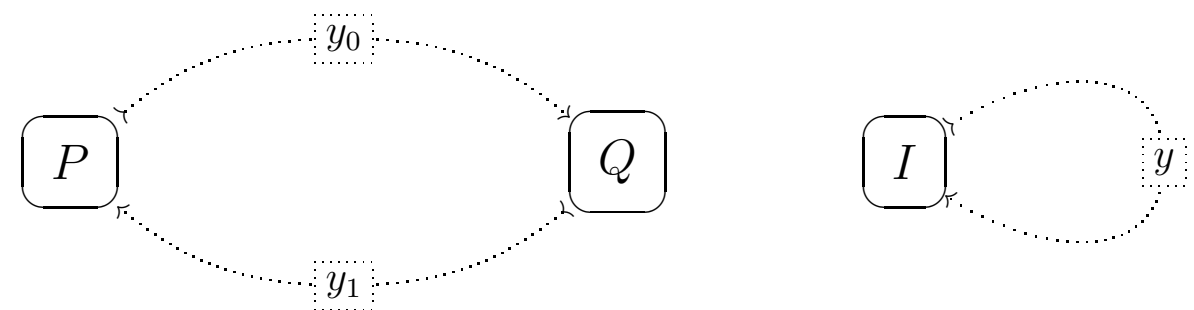

In process $\llbracket P \mid Q \rrbracket$, imagine the situation, where both receivers for $y_{0}$ and $y_{1}$ have input the matching request, and afterwards successfully tested their own choice-lock: here, both have to wait for their respective sender's choice-lock to become available again, but neither of them will do, so both receivers remain blocked forever. This symmetric cyclic-wait situation is very similar to the classical 'dining philosophers' problem [RL94], where several (in our case: two) processes compete for mutually exclusive access to forks (locks).

\footnotetext{
${ }^{3}$ Note that the presence of output-guarded replication in the source language would not invalidate this result, since in the encoding, this construct would be translated by mentioning a lock that always carries $t$ due to the lack of competitors, thus invalidating condition (3) in the proof.
} 
In process $\llbracket I \rrbracket$, the sender's request on $y$ could be consumed by the competing receiver branch, which results in a deadlock situation, because the receiver would try to test the same lock twice, which is impossible.

Breaking the symmetry In Distributed Computing, one method to resolve cyclic dependencies among processes is by using time-outs or probabilistic algorithms for the attempt to acquire some lock. Then, however, we face the problem of infinite loops, such that randomized solutions are not 'reasonable' [Pal97], although it is known that solutions exist that guarantee progress with probability 1 [RL94]. If, in such cases, we assume fair execution schedulers, then divergence is not harmful anymore, as long as there is no danger for live-locks.

Another method, known from the distributed implementation of concurrent languages, is exploiting a total order among the threads in the system by, for example, always choosing the lock of the smaller thread first [Ber80, BS83, Kna93], when required to make a choice. Then, the above symmetric cyclic-wait situation is immediately prevented since both receivers choose the same thread, i.e. lock, as the first to interrogate. Note also that under a total order assumption symmetric networks according to [Pal97] do not exist.

In the following subsections, we adapt the methods of randomization (see §4.1) and total ordering of threads (see $\S 4.2$ and $\S 4.3$ ) to our case of encoding mixed choice into the asynchronous $\pi$-calculus, and we evaluate their properties.

\subsection{A randomized solution}

Randomization means removing determinism from an algorithm and adding randomly possible computation paths. In our case, instead of choosing a fixed order for testing the locks as in Figure 5, we might allow ourselves to test them nondeterministically in either order and allow first-phase locks to be given back (cf. [RL94]). Of course, in our target language we cannot trivially write down "either receive from the second lock, or resend on the first lock", because in order to do so, we would need a mixed choice construct. Note that we cannot use internal choice either, because it would only delay potential deadlocks, which arise when the internal decision favors the branch "waiting for the second lock".

In Figure 6, we model a randomized solution based on the encoding in Figure 5 by only supplying a new clause for receivers. ${ }^{4}$ We use a local state, implemented as a mutex channel $s$ that carries a $\operatorname{tag}^{5}$ (and a boolean value) that tells, whether none ( $\left.\operatorname{tag} N\right)$, the local $(\operatorname{tag} \mathrm{L})$ or the remote $(\operatorname{tag} \mathrm{R})$ lock are currently held by the receiver. The tag-information, initially $\mathrm{N}$ (w.l.o.g. with value $\mathrm{f}$ ), is supplied by two processes, called lock-checkers, waiting at $l c l$ and rmt, which try to get hold of the local lock $l$ and remote lock $r$, respectively. After grabbing a lock, these processes need to read the current state: if the complementary lock is

\footnotetext{
${ }^{4} \mathrm{~A}$ similar solution is used in the implementation of receivers in the join-calculus [FG96].

${ }^{5}$ We use this special syntax for the sake of readability; since we only need 3 different tags, we can easily simulate them by 2 boolean tags and use the corresponding if- and test-expressions for matching.
} 


$$
\begin{aligned}
& \llbracket y ?[\tilde{x}] . P \rrbracket_{l} \stackrel{\text { def }}{=}(\boldsymbol{\nu} b)\left(b ![] \mid b ?^{*}[] . y ?[r, a, \tilde{x}] .\right. \\
& (\boldsymbol{\nu} s, l c l, r m t, r n d, b t h)\left(\quad l c l ?^{*}[] . l ?\left[b_{L}\right] . s ?[t a g, b]\right. \text {. } \\
& \text { if } \operatorname{tag}=\mathrm{R} \text { then } b t h !\left[b_{L}, b\right] \mid s ![\mathrm{N}, \mathrm{f}] \text { else } s !\left[\mathrm{L}, b_{L}\right] \mid r n d ![] \\
& r m t ?^{*}[] . r ?\left[b_{R}\right] . s ?[\operatorname{tag}, b] \text {. } \\
& \text { if } t a g=\mathrm{L} \text { then } b t h !\left[b, b_{R}\right] \mid s ![\mathrm{N}, \mathrm{f}] \text { else } s !\left[\mathrm{R}, b_{R}\right] \mid r n d ![] \\
& r n d ?^{*}[] . s ?[\operatorname{tag}, b] .(s ![\mathrm{N}, \mathrm{f}] \mid \text { if } \operatorname{tag}=\mathrm{L} \text { then } l ![b] \mid l c l ![] \text { else } \\
& \text { if } \operatorname{tag}=\mathrm{R} \text { then } r ![b] \mid r m t ![] \text { else } \mathbf{0} \text { ) } \\
& b t h ?\left[b_{L}, b_{R}\right] . \text { if } \quad b_{L} \wedge b_{R} \text { then } l ![\mathrm{f}]|r ![\mathrm{f}]| a ![\mathrm{t}] \mid \llbracket P \rrbracket \text { else } \\
& \text { if } b_{L} \quad \text { then } l ![\mathrm{t}]|r ![\mathrm{f}]| a ![\mathrm{f}] \mid b ![] \text { else } \\
& \text { if } \quad b_{R} \text { then } l ![\mathrm{f}]|r ![\mathrm{t}]| y ![r, a, \tilde{x}] \\
& \text { else } \quad l ![\mathrm{f}]|r ![\mathrm{f}]| a ![\mathrm{f}] \\
& l c l ![]|r m t ![]| s ![\mathrm{N}, \mathrm{f}]))
\end{aligned}
$$

Figure 6: Randomized $\mathbb{S}^{\text {sep }} \rightarrow \mathbb{T}$ for use as $\mathbb{S}^{\text {mix }} \rightarrow \mathbb{T}$

already held, then the two lock values are passed on to the analyzer process waiting at bth and the state $s$ is initialized; otherwise, the state $s$ is appropriately updated to announce success for getting the current lock and, in addition to this announcement, a randomizer process at $r n d$ is started that competes with the lock-checkers for reading the state. If the randomizer succeeds in reading the state, it resets the state and resends the lock, while restarting the corresponding lock-checker. If both lock-checkers succeed reading the state without the randomizer interfering, then $s$ is left with its initial value and is finally consumed by the active randomizer to terminate the system by resetting the state without restarting any of the lock-checkers and without restarting the randomizer itself. Note that after restarting the whole receiver at $b$ in the case of local success $\left(b_{L}=\mathrm{t}\right)$ and remote failure $\left(b_{R}=\mathrm{f}\right)$, a new state will be created, when a new request on $y$ arrives.

Evaluation As the encoding for separate choice, the randomized encoding for mixed choice in Figure 6 is uniform since restriction and parallelism are encoded purely compositionally. The randomized encoding is deadlock-free due to the ever present possibility of backing out, when a second-phase lock is not available: all receivers on the state channel-both lockcheckers and the randomizer - have equal priority, so in case of a potential deadlock, the randomizer can help, since it is triggered, whenever a non-trivial state is set. (Note that, again, we only use channels of reliable type: $b, l c l$, rmt, and $r n d$, are replicated, $b t h$ is linear, and $s$ is mutex. So we can apply Kobayashi's type system for the proof.) 
However, the encoding is not divergence-free, since the randomizer introduces potentially infinite loops. Yet, under fair execution, divergence would be prevented with probability 1. Furthermore, the encoding is livelock-free - again due to the ever present possibility of backing out: whenever the randomizer is starting to loop by continuously trying to reset the state after one of the lock-checkers has proceeded, we know that there is always a second lock-checker ready and willing to interfere. The liveness of the lock-checker rests on the fact that lock messages are correctly used according to the obligations of mutex channels, which have the important property to become available again and again.

\subsection{A 'bakery' algorithm}

The $\pi$-calculus itself does not directly provide total ordering information as required for modelling the choice protocols as used in the distributed implementations mentioned in the introduction of $\S 4$. However, we may program a number server, which can be interrogated to dynamically provide unique global numbers when required, reminiscent of Lamport's bakery algorithm (cf. [Lam74]). Natural numbers as well as comparison operators can be easily encoded in the $\pi$-calculus [Mil93]. For convenience, we add them explicitly: let if $n<m$ then $P_{1}$ else $P_{2}$ be a comparison operator, where $m$ and $n$ become integers (in $\mathbb{N}$ ), and let now $x, z \in \mathbf{V}:=\mathbf{N} \cup \mathbb{B} \cup \mathbb{N}$ in $\mathbb{T}$ (with straightforward extensions to the type system).

A single globally accessible channel $c$ suffices to implement a bakery algorithm for our purposes. However, this channel must not be accessible by external processes, which might possibly violate the numbering mechanism. Therefore, an encoding according to this programming scheme (see Figure 7) must appear as a two-level definition: an internal compositional encoding (fully compositional according to [Pal97]) that is parameterized on the global channel, equipped with a top-level context that protects the global counting mechanism and restricts access to the translations of the original processes. At the top-level, $c$ is initialized with some integer value and passed on as a parameter to the inner compositional encoding $\llbracket \rrbracket^{c}$. Essentially, $c$ is only used, when a thread enters a choice point (our 'bakery'). There, it is dynamically equipped with a globally unique number $n$. Immediately incrementing the counter, this number is transmitted as an additional parameter of the threads' send requests and used later on in the protocol of the receivers. Figure 7 shows the corresponding variant of the protocol for separate choice, now adapted to mixed choice using two different strands of actions based on the ordering of the locks.

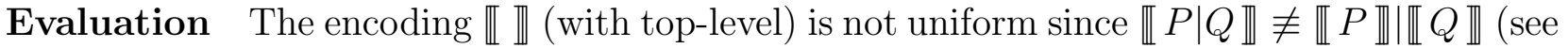
also Appendix A), whereas the mere inner encoding $\llbracket \rrbracket^{c}$ is uniform. The encoding is deadlockfree, since we (1) prevent cyclic waiting on locks by using a variant of the bakery algorithm, and (2) deal with 'incestuous' communication by checking equality $n=m$ of the request's id's, such that an unintended send-request is resent and the receiver's loop is restarted. Knabe's graphbased proof sketch [Kna93] for deadlock-freedom of his implementation could be adapted to 


$$
\begin{aligned}
& \llbracket P \rrbracket \quad \stackrel{\text { def }}{=}(\boldsymbol{\nu} c)\left(c ![42] \mid \llbracket P \rrbracket^{c}\right) \\
& \llbracket P_{1}\left|P_{2} \rrbracket^{c} \stackrel{\text { def }}{=} \llbracket P_{1} \rrbracket^{c}\right| \llbracket P_{2} \rrbracket^{c} \\
& \llbracket(\boldsymbol{\nu} x) P \rrbracket^{c} \quad \stackrel{\text { def }}{=}(\boldsymbol{\nu} x) \llbracket P \rrbracket^{c} \\
& \llbracket \sum_{i \in I} \pi_{i} . P_{i} \rrbracket^{c} \stackrel{\text { def }}{=} c ?[n] .\left(c ![n+1] \mid(\boldsymbol{\nu} l)\left(l ![\mathrm{t}] \mid \prod_{i \in I} \llbracket \pi_{i} . P_{i} \rrbracket_{n, l}^{c}\right)\right) \\
& \llbracket y ![\tilde{z}] . P \rrbracket_{n, l}^{c} \stackrel{\text { def }}{=}(\boldsymbol{\nu} a)\left(y ![n, l, a, \tilde{z}] \mid \text { test } a \text { then } \llbracket P \rrbracket^{c} \text { else } \mathbf{0}\right) \\
& \llbracket y ?[\tilde{x}] . P \rrbracket_{n, l}^{c} \stackrel{\text { def }}{=}(\boldsymbol{\nu} b)\left(b ![] \mid b ?^{*}[] .\right. \\
& y ?[m, r, a, \tilde{x}] . \\
& \text { if } \quad n=m \text { then }(y ![m, r, a, \tilde{x}] \mid b ![]) \text { else } \\
& \text { if } \quad n<m \\
& \text { then test } l \\
& \text { then test } r \\
& \text { then } l ![\mathrm{f}]|r ![\mathrm{f}]| a ![\mathrm{t}] \mid \llbracket P \rrbracket^{c} \\
& \text { else } l ![\mathrm{t}]|r ![\mathrm{f}]| a ![\mathrm{f}] \mid b ![] \\
& \text { else } l ![\mathrm{f}] \mid y ![m, r, a, \tilde{x}] \\
& \text { else test } r \\
& \text { then test } l \\
& \text { then } l ![\mathrm{f}]|r ![\mathrm{f}]| a ![\mathrm{t}] \mid \llbracket P \rrbracket^{c} \\
& \text { else } l ![\mathrm{f}]|r ![\mathrm{t}]| y ![m, r, a, \tilde{x}] \\
& \text { else } r ![\mathrm{f}]|a ![\mathrm{f}]| b ![]) \\
& \llbracket y ?^{*}[\tilde{x}] . P \rrbracket^{c} \stackrel{\text { def }}{=} y ?^{*}[n, r, a, \tilde{x}] \text {.test } r \text { then } r ![\mathrm{f}]|a ![\mathrm{t}]| \llbracket P \rrbracket^{c} \text { else } r ![\mathrm{f}] \mid a ![\mathrm{f}]
\end{aligned}
$$

Figure 7: A 'bakery' solution for $\mathbb{S}^{\text {mix }} \rightarrow \mathbb{T}$

the current setting. See Appendix B for a discussion on an extension of Kobayashi's typing system [Kob97] to cope with the encoding.

Unfortunately, the encoding is not quite divergence-free due to the way we avoid deadlocks in the case of 'incestuous' self-communication in the $n=m$ clause: a sender's request may be re-consumed again and again. Yet, the encoding is still livelock-free, since for every enabled matching competitor of an incestuous pair of branches it is always, i.e. again and again, possible to stop the self-communication. 


\subsection{A 'practical' solution}

The main theme in this subsection is the approach of changing the source and target languages of the choice encodings to reflect some phenomena that occur in distributed implementations. It turns out that the source language can be restricted to shrink the number of programs that are difficult to cope with. On the other hand, we propose an extension of the target language that handles total ordering at an abstract level.

Dealing with 'incestuous' self-communication A quick solution for the above unwanted divergence defines the source language such that 'incestuous' self-communication in mixed choices is allowed, similar to the self-communication in the output prefixes of Milner's synchronous $\pi$-calculus, as observed by Bellin and Scott [BS94]. So, if we trigger the continuation processes in the $n=m$ clause of the receiver's protocol in the case that the local lock (which is then the same as the remote lock) can be successfully tested, as in

$$
\text { if } n=m \text { then ( test } l \text { then } l ![\mathrm{f}]|a ![\mathrm{t}]| \llbracket P \rrbracket \text { else } l ![\mathrm{f}] \mid a ![\mathrm{f}] \text { ) else } \ldots
$$

then we actually get a (still not uniform, but) deadlock- and divergence-free encoding.

In contrast, Knabe's implementation [Kna93] models a channel as a process that collects send and receive requests on queues. It only then considers two matching requests as candidates to enter the communication protocol, if they belong to different choices. Such implementations are not uniform (see Appendix A), but deadlock- and divergence-free.

Another practically motivated solution is due to the observation that, in distributed systems, it is often the case that receivers are localized, i.e. on each channel there is only one receiver waiting. This can be exploited for both implementation and reasoning; see the work on the join-calculus [FG96, Ama97], where such forms of locality are guaranteed either syntactically or by a simple type system, and also the work on linear receptiveness [San97]. We also profit from a unique-receiver property: 'incest' can then be avoided without divergence by simply throwing away the critical send-request; no other receiver could be waiting for it. This is the approach taken in Figure 8 (see the line for $n=m$ ).

Bakery primitive Here, we recall the idea of deriving a total order among threads (lightweight processes) from some distribution order among the nodes and processors and processes' creation id's for finer identification [Ber80, BS83, Kna93]. For the $\pi$-calculus, we choose an abstract view, that captures the idea syntactically. Let us assume an extended target language $\pi_{\mathrm{a}}^{\kappa}$ with a binding primitive $(\boldsymbol{\kappa} n) P$ for creating totally ordered identifiers $n$ in process $P$, then our bakery algorithm can be programmed in a 'uniform' way without the need of a top-level: in the encoding of choice in Figure 8 (see line 3 ) the actual identity of $n$ is not important - it only matters that every pair $n, m$ of different identifiers is ordered.

With the 'bakery primitive' in the target $\left(\pi_{\mathrm{a}}^{\kappa}\right)$ and a unique-receiver property in the source $\left(\pi_{s}^{\mathrm{mix}, 1}\right)$, we get the encoding in Figure 8. It is similar to Knabe's distributed implementation [Kna93], but replaces channel managers by assuming unique receivers. 


$$
\begin{aligned}
& \llbracket P_{1}\left|P_{2} \rrbracket \quad \stackrel{\text { def }}{=} \llbracket P_{1} \rrbracket\right| \llbracket P_{2} \rrbracket \\
& \llbracket(\boldsymbol{\nu} x) P \rrbracket \stackrel{\text { def }}{=}(\boldsymbol{\nu} x) \llbracket P \rrbracket \\
& \llbracket \sum_{i \in I} \pi_{i} . P_{i} \rrbracket \quad \stackrel{\text { def }}{=}(\boldsymbol{\kappa} n)(\boldsymbol{\nu} l)\left(l ![\mathrm{t}] \mid \prod_{i \in I} \llbracket \pi_{i} \cdot P_{i} \rrbracket_{n, l}\right) \\
& \llbracket y ![\tilde{z}] . P \rrbracket_{n, l} \stackrel{\text { def }}{=}(\boldsymbol{\nu} a)(y ![n, l, a, \tilde{z}] \mid \text { test } a \text { then } \llbracket P \rrbracket \text { else } \mathbf{0}) \\
& \llbracket y ?[\tilde{x}] . P \rrbracket_{n, l} \stackrel{\text { def }}{=}(\boldsymbol{\nu} b)\left(b ![] \mid b ?^{*}[] .\right. \\
& y ?[m, r, a, \tilde{x}] . \\
& \text { if } \quad n=m \text { then } b ![] \text { else } \\
& \text { if } \quad n<m \\
& \text { then test } l \\
& \text { then test } r \\
& \text { then } l ![\mathrm{f}]|r ![\mathrm{f}]| a ![\mathrm{t}] \mid \llbracket P \rrbracket \\
& \text { else } l ![\mathrm{t}]|r ![\mathrm{f}]| a ![\mathrm{f}] \mid b ![] \\
& \text { else } l ![\mathrm{f}] \mid y ![m, r, a, \tilde{x}] \\
& \text { else test } r \\
& \text { then test } l \\
& \text { then } l ![\mathrm{f}]|r ![\mathrm{f}]| a ![\mathrm{t}] \mid \llbracket P \rrbracket \\
& \text { else } l ![\mathrm{f}]|r ![\mathrm{t}]| y ![m, r, a, \tilde{x}] \\
& \text { else } r ![\mathrm{f}]|a ![\mathrm{f}]| b ![]) \\
& \llbracket y ?^{*}[\tilde{x}] . P \rrbracket \quad \stackrel{\text { def }}{=} y ?^{*}[n, r, a, \tilde{x}] \text {.test } r \text { then } r ![\mathrm{f}]|a ![\mathrm{t}]| \llbracket P \rrbracket^{c} \text { else } r ![\mathrm{f}] \mid a ![\mathrm{f}]
\end{aligned}
$$

Figure 8: $\pi_{s}^{\mathrm{mix}, 1} \rightarrow \pi_{\mathrm{a}}^{\kappa}$

Evaluation The encoding in Figure 8 gets rid of the problem of possible self-communication by exploiting uniqueness, and is deadlock-free - and also divergence-free-by exploiting the inherent total-order. Note that such encodings do not preserve the symmetry of networks, as necessary for Palamidessi's impossibility argumentation, because the target language is intrinsically asymmetric due to the totally ordering 'bakery' primitive. Since such languages allow for fully compositional encodings of mixed-guarded choice, while the standard (symmetric) asynchronous $\pi$-calculus only allows for 'semi-compositional' encodings, this can be interpreted as a separation between symmetric and asymmetric calculi. 


\section{$5 \quad$ Full abstraction}

An encoding is often considered correct only if it is fully abstract with respect to some notions of equivalence in the source and target language, meaning that these notions are to be preserved and reflected by the encoding. In $\S 5.1$, we recall the problem of gradual commitments, e.g. known from the encoding of input-guarded choice, and its consequences on full abstraction. In $\S 5.2$, we summarize a few observations that highlight the inherent problems of stating full abstraction properties - with respect to barbed bisimulation - in the presence of outputguards. In $§ 5.3$, we state a rather restricted full abstraction result, which can nevertheless be interpreted as: 'programming with choice' is implemented correctly.

\subsection{Gradual commitments}

The encoding of input-guarded choice in Figure 4 is not fully abstract with respect to weak asynchronous bisimulation $\approx_{a}$. This can be shown by using a simple counter-example that turns atomic commitments of the source into so-called gradual commitments in the target.

Fact 5.1.1. Let $S=\overline{y_{2}} \mid y_{1} \cdot \overline{p_{1}}+y_{2} \cdot \overline{p_{2}} \in \mathbb{S}^{\text {inp }}$. Then $S \not \varkappa_{\mathrm{a}} \llbracket S \rrbracket$.

Intuitively, at the source-level, when a send-request is consumed by some receiver, this action also resolves the choice atomically. At the target-level, the consumption of the message $\overline{y_{2}}$ merely means that this receiver has started its choice protocol; it is not yet decided, in general, that this receiver will win - a message $\overline{y_{1}}$, which may be supplied by the context and is actually considered by the notion of weak asynchronous bisimulation, might join the competition on its way before the choice-lock is actually read. At this stage, it is only clear that this choice will eventually be resolved and, unless that happens, the messages losing in this game will not become available to other receivers in other choices.

Using the above example, we proved that the encoding of Figure 4 neither preserves nor reflects weak asynchronous bisimulation [NP96]. However, this encoding is nevertheless fully abstract with respect to an asynchronous version of coupled simulation [PS92], Note that we can also manipulate the encoding to become fully abstract with respect to weak asynchronous bisimulation, but we only managed to do that by introducing divergence [NP96], which we are meant to avoid, here, in order to satisfy Palamidessi's criteria.

As the encodings for separate and mixed choice in this paper are built on the same idea of gradual commitments, we cannot expect to do better, so also have to cope with gradual commitments in these cases and, therefore, weak bisimulation is not promising success. However, even aiming at coupled simulation turns out to be problematic since there are other problems that are inevitable in the presence of output guards. These are best explained using the notion of barbs. 


\subsection{On barbed bisimulation}

An encoding may be considered sufficiently adequate if it is fully abstract with respect to barbed bisimulation [MS92], which concentrates on the correspondence between the reductions of source and target terms. In addition, barbed bisimulation only requires the correspondence between the terms' barbs - an observation predicate that captures the immediate communication capabilities, e.g. input, output, or just convergence - without taking potentially nasty contexts into account. Barbed congruence is capturing context-sensitive behavior by means of a universal quantification on top of barbed bisimulation.

As usual, there are strong and weak versions of barbed bisimulation. It is the weak case, which is interesting for reasoning about the correctness of encodings since, in general, one high-level reduction corresponds to several low-level reductions. We only introduce the weak case, and we only consider output barbs, since they correspond to asynchronous bisimulation [ACS98], which is useful for reasoning about choice encodings [NP96].

We first recall a few definitions, for which we let $S_{y}$ be a sender on $y$ : depending on the underlying language $\mathbb{P}$ being either of the $\mathbb{S}^{\Sigma}$ or $\mathbb{T}$, this is just a message $\bar{y}\langle\tilde{z}\rangle$ (for $\mathbb{T}$, $\mathbb{S}^{\text {inp }}$ ), or a choice term $R+y ![\tilde{z}] . Q$ with an output (prefix) on $y$ sitting inside (for $\mathbb{S}^{\text {sep }}, \mathbb{S}^{\text {mix }}$ ).

Definition 5.2.1 (Barbed bisimulation). Let $P \in \mathbb{P}$. Then:

- $P \downarrow_{y}$ iff $P \equiv(\boldsymbol{\nu} \tilde{x})\left(\dot{P} \mid S_{y}\right)$ for $y \notin \tilde{x}$, and $P \Downarrow_{y}$ iff $P \Rightarrow P^{\prime} \downarrow_{y}$ for some $P^{\prime}$.

- A relation $\mathcal{R}$ is a barbed bisimulation, if $(P, Q) \in \mathcal{R}$ implies

- If $P \rightarrow P^{\prime}$, then there is $Q \Rightarrow Q^{\prime}$ with $\left(P^{\prime}, Q^{\prime}\right) \in \mathcal{R}$.

- If $P \Downarrow_{y}$, then $Q \Downarrow_{y}$.

and vice versa. Two processes are barbed bisimilar, written $P \dot{\approx} Q$, if there is some barbed bisimulation with $(P, Q) \in \mathcal{R}$.

We use the notation $S \Downarrow_{N}$ to denote $\forall x \in N \subseteq \mathbf{N}: S \Downarrow_{x}$, and $S \Downarrow_{\emptyset}$ for $\nexists x \in \mathbf{N}: S \Downarrow_{x}$.

Note If we do not take into account observing contexts - as it is the case when we concentrate on barbed bisimulation - then we can no longer observe gradual commitments. The reason is that, in the translation, barbs do not disappear during gradual commitments, but only when the last committing step - the successful testing of the lock - is performed. Thus, for the above counter-example of Fact 5.1.1, we have $S \dot{\approx} \llbracket S \rrbracket$, so we cannot use it to prove a negative result for input-guarded choice. However, if we get positive full abstraction results for our choice encodings with respect to barbed bisimulation - as it is the case for input-guarded choice, for the others see below - then every attempt to strengthen the result by taking contexts into account that potentially contain messages, will suffer from the problem of gradual commitments and require some 'coupled' adaptation of the involved bisimulation (Fournet and Gonthier have recently proposed a range of such [FG98]). 
In the following, whenever we do not exactly specify the encoding function $\llbracket \rrbracket$, we use it as a placeholder for all choice encodings in this paper. In order to approach full abstraction with respect to barbed bisimulation, we check encodings for (1) the correspondence of barbs and (2) the correspondence of reductions in terms and their translations. Whereas barbed correspondence is straightforward, operational correspondence will require some machinery.

Lemma 5.2.2 (Barbed correspondence). Let $S \in \mathbb{S}^{\Sigma}$. Then: $S \Downarrow_{y}$ iff $\llbracket S \rrbracket \Downarrow_{y}$.

Proof. Immediate for $\Sigma \in\{$ inp $\}$. Straightforward for $\Sigma \in\{$ sep, mix $\}$ by looking at the encoding for the case of choice into parallel composition and synchronous outputs. The direction "only if" holds since each of the channels $l, r, a, b$ that are introduced by $\llbracket \rrbracket$ is initially restricted, so the only observable actions are on high-level channels.

\section{Problem 1: Invalid outputs}

While the encoding of input-guarded choice satisfies a nice correspondence between reductions in terms and their translations (expressed as a pair of coupled asynchronous simulations), encodings that need to consider output-guards in choices cause severe problems in this case, as depicted in Figure 9: after simulating the choice for branch $k$ with a sequence of low-level steps there is some active non-chosen 'garbage' $G_{k}$ (indicated as the underlined remainder of the encoded choice after choosing $k$ ) in the system that is running in parallel with the intended encoding $\llbracket Q^{\prime} \mid P_{k}^{\prime} \rrbracket$ of the continuation of the communication partners. For the encoding of input-guarded choice, we found that an asynchronous observation principle [HT92, ACS98] yields an appropriate notion of equivalence $\approx_{\mathrm{a}}$ since it allows us to garbage-collect processes that do nothing else than eventually resending every message that they consume, so $G_{k} \approx_{\mathrm{a}} \mathbf{0}$ holds in this case. With output-guards, however, non-chosen branches may still perform asynchronously visible outputs, which-according to their lock-information - are not valid, so $G_{k}$ exhibits too much observable behavior.

Let us look at the details for $\Sigma \in\{\operatorname{sep}\}$. Every visible activity of a term $T$ that is reachable via reduction from some translation $\llbracket S \rrbracket$ is necessarily on some high-level channel. The reason is that the low-level channels $l, r, a, b$ are introduced under restriction, which cannot be opened up by reductions, but only by output. So, by analysis of Figure 5 :

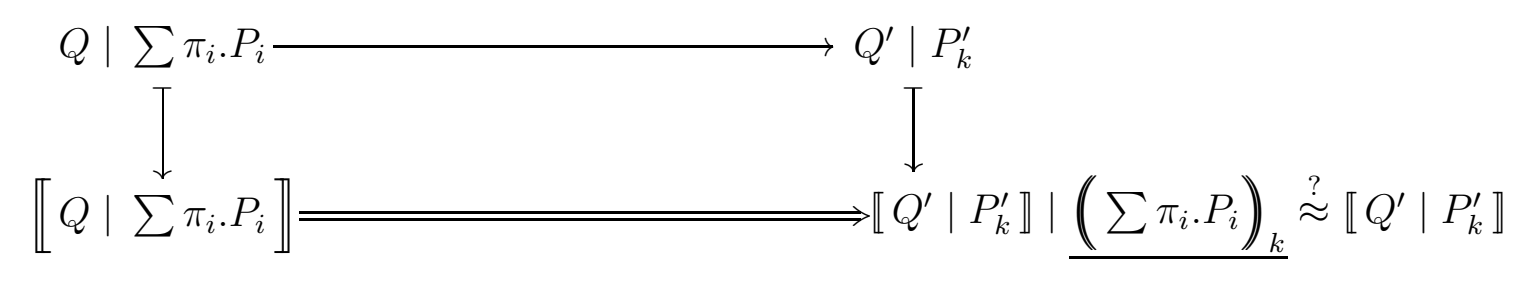

Figure 9: Simulation of a choice reduction, committing to branch $k$ 
if $\llbracket S \rrbracket \Rightarrow T \downarrow_{y}$, then $T \equiv(\boldsymbol{\nu} l, a, \tilde{x})(y ![l, a, \tilde{z}] \mid \dot{T})$ for some $\dot{T}$ and $l, a, \tilde{x}, \tilde{z}$ with $\tilde{x} \subseteq \tilde{z}$.

Observe that, according to $\dot{T}$, it can happen that the lock channel $l$ will eventually signal to the receiver (of the names $l, a, \tilde{z}$ along $y$ ) that the state of the sender's choice has already been resolved, so that the previous output on $y$ was actually a zombie - an 'invalid' output that does not correspond to the source level behavior. Note that for the above example $G_{k}$, no output at all is valid. Consequently, weak barbed correspondence for the encoding of separate choice (and also for mixed choice, see below) does not hold.

In order to 'bend' the notion of barb for dealing with separate choices, we introduce a tailored variant that captures the observation of 'valid' outputs in this case. Note that, in the above analysis of outputs on high-level channels, for every such output, we can always immediately find a message on the mutex channel $l$ that is mentioned in the output:

Lemma 5.2.3. Let $S \in \mathbb{S}^{\mathrm{sep}}$ and $\llbracket S \rrbracket \Rightarrow T \downarrow_{y}$. Then $T \equiv(\boldsymbol{\nu} l, a, \tilde{x})(y ![l, a, \tilde{z}]|l ![\mathrm{b}]| \hat{T})$ for some $\hat{T} \in \mathbb{T}, \mathrm{b} \in \mathbb{B}$, and $l, a, \tilde{x}, \tilde{z}$ with $\tilde{x} \subseteq \tilde{z}$.

Proof. By structural analysis of the encoding, emphasizing the case for output prefix.

Consequently, we can syntactically check, whether a low-level output on some high-level channel is valid $(b=t)$ or not $(b=f)$. Using this lemma, we define in $\mathbb{T}$ the notion of a tailored $\Sigma$-barb for $\Sigma \in\{$ sep $\}$ as the existence of an output with 'witnessed validity':

$$
T \downarrow_{y}^{\text {sep }} \quad \text { iff } \quad T \equiv(\boldsymbol{\nu} l, a, \tilde{x})(y ![l, a, \tilde{z}]|l ![\mathrm{t}]| \hat{T})
$$

for some $\hat{T}$, and $T \Downarrow_{y}^{\text {sep }}$ if $T \Rightarrow T^{\prime} \downarrow_{y}^{\text {sep }}$ for some $T^{\prime}$. Following the standard definitional path, we may provide a straightforward notion of barbed bisimulation based on $\Sigma$-barbs.

Definition 5.2.4 ( $\Sigma$-barbed bisimulation). A relation $\mathcal{R}$ is a $\Sigma$-barbed bisimulation, if $(P, Q) \in \mathcal{R}$ implies

- If $P \rightarrow P^{\prime}$, then there is $Q \Rightarrow Q^{\prime}$ with $\left(P^{\prime}, Q^{\prime}\right) \in \mathcal{R}$.

- If $P \Downarrow_{y}^{\Sigma}$, then $Q \Downarrow_{y}^{\Sigma}$.

and vice versa. Two processes are called $\Sigma$-barbed bisimilar, written $P \dot{\bowtie}$, if there is some $\Sigma$-barbed bisimulation with $(P, Q) \in \mathcal{R}$.

Now, the desired property for non-chosen branches (according to Figure 9) holds.

Lemma 5.2.5 (Garbage). For $\llbracket \rrbracket$ on $\left.\mathbb{S}^{\mathrm{sep}} \rightarrow \mathbb{T}:\left(\sum \pi_{i} . P_{i}\right)\right)_{k} \dot{\bowtie} \mathbf{0}$

Proof. Immediate, since $\left(\sum \pi_{i} \cdot P_{i}\right)_{k} \Downarrow_{\emptyset}$. 
We give two different ways of relating reductions in the source and target. The first makes explicit gradual commitments by stating that target descendants of translations always correspond to a state that is potentially in between two state in the source.

Lemma 5.2.6 (Operational correspondence - I). Let $S \in \mathbb{S}^{\mathrm{sep}}$.

1. If $S \rightarrow S^{\prime}$, then

there is $\llbracket S \rrbracket \Rightarrow T$ with $T \dot{\bowtie} \llbracket S^{\prime} \rrbracket$.

2. If $\llbracket S \rrbracket \Rightarrow T$, then either $\llbracket S \rrbracket \dot{\approx} T$ or

there are $S \Rightarrow S_{1} \rightarrow S_{2}$ and $\llbracket \rrbracket \Rightarrow T_{1} \Rightarrow T \Rightarrow T_{2}$ with $T_{i} \dot{\bowtie} \llbracket S_{i} \rrbracket$.

The following formulation emphasizes that gradual commitments are not noticed by barbed bisimulation. For this purpose, we use the abbreviation $P \stackrel{\bullet}{\longrightarrow} P^{\prime}$ to denote that $P \rightarrow P^{\prime}$ and also $P \dot{\approx} P^{\prime}$, while we use $P \stackrel{\bullet}{\Longrightarrow} P^{\prime}$ to denote that $P=P_{0} \stackrel{\bullet}{\longrightarrow} P_{1} \stackrel{\bullet}{\longrightarrow} \ldots \stackrel{\bullet}{\longrightarrow} P_{n}=P^{\prime}$.

Lemma 5.2.7 (Operational correspondence - II). Let $S \in \mathbb{S}^{\mathrm{sep}}$.

1. If $S \rightarrow S^{\prime}$,

then there is $\llbracket S \rrbracket \stackrel{\bullet}{\longrightarrow} T \rightarrow^{2} T^{\prime}$ with $T^{\prime} \dot{\bowtie} \llbracket S^{\prime} \rrbracket$.

2. (a) If $\llbracket S \rrbracket \stackrel{\doteq}{\Longrightarrow} \rightarrow T^{\prime \prime}$ with $T \dot{\neq} T^{\prime \prime}$,

then there are $S \Rightarrow S^{\prime}$ and $T^{\prime \prime} \rightarrow T^{\prime}$ with $T^{\prime} \dot{\bowtie} \llbracket S^{\prime} \rrbracket$.

(b) If $\llbracket$ $S \rrbracket \Rightarrow T$,

then either $\llbracket S \rrbracket \dot{\approx} T$ or there is $S \Rightarrow S^{\prime}$ with $T \dot{\bowtie} \llbracket S^{\prime} \rrbracket$.

Proof. (Sketch) By induction on the reductions in source and target. The problems here are mostly notational and can be handled by annotation techniques as in [NP96].

(1) By "following our nose", we first perform one reduction (internal) for triggering the required input-guard loop and one reduction for consuming the respective send-request, while we remain in the same equivalence class. Then, we perform the committing steps to test the two locks. Note here that either of the first or the second test may be be committing, depending on $S$ : if there are no competitors for the sender-lock, then the first test (for the receiver-lock) is already committing, but this is not true in general.

(2a) We need to take into account that arbitrary irrelevant reductions may take place in the target, but once we leave the equivalence class, this corresponds to some high-level reduction. By structural analysis, we decompose $P$ into the part relevant for the last reduction leading to it. Note that $\dot{\bowtie}$ includes $\dot{\approx}$, so the unnecessary initial reductions do not harm and can be kept separate.

(2b) We apply (2a) repeatedly according to the shape of $\llbracket S \rrbracket \Rightarrow P$. 


\section{Open problem (Full abstraction). Let $S \in \mathbb{S}^{\mathrm{sep}}$. Then: $S_{1} \dot{\approx} S_{2}$ iff $\llbracket S_{1} \rrbracket \dot{\bowtie} \llbracket S_{2} \rrbracket$.}

We have not yet worked out the details for the proof, but we believe that this full abstraction result actually holds and can be proven using the correspondence lemmas 5.2.7 and 5.2.2.

Since the notion of $\Sigma$-barbed bisimulation is rather artificial and tailored for our application, we do not expect a wider applicability for it. Nevertheless, we think that $\Sigma$-barbs are instructive for a better understanding of encodings of choice with output-guards. To finalize this semi-formal study, we point out two more problems that deal with the case of mixed choices and with the investigation of congruence properties.

\section{Problem 2: Reversed testing and mixed choice}

The above definition of $\Sigma$-barbs was possible for $\Sigma \in\{\operatorname{sep}\}$ since in the encoding of Figure 5 sender-locks are always checked in the second place: in contrast to receiver-locks, which might not be available for a couple of reductions, testing a sender's lock (always after having successfully tested the receiver's lock) immediately causes its re-set (Figure 5). If we changed the order of checking locks in $\llbracket \rrbracket: \pi_{\mathrm{s}}^{\mathrm{sep}} \rightarrow \mathbb{T}$ to test $r$ then test $l$ then $\ldots$, there would be situations, where the required mutex message is not available.

For example, in $\llbracket u ?[x] . K+y ?[x] . P \mid y ![z] . Q+w ![v] . R \rrbracket$ let the sender and receiver on $y$ have exchanged the send-request and the receiver checked the sender's lock $r$. In that situation, it cannot be observed directly, i.e. from the syntax of the (encoded) term, whether the possible output on $w$ is valid, or not. It is valid, because its choice has not yet been resolved in favor of $y$ (since the receivers' lock $l$ has not yet been checked), but neither is the necessary lock $r$ available (since it is currently held by the receiver on $y$ ), nor can a state be reached by reduction, where the lock $r$ is available again, without committing to the communication on $y$ and turning the senders' lock $r$ to $\mathrm{f}$; the only way to detect the validity of the output on $w$ would be by supplying a message on $u$ from the outside and observing that the communication on $u$ could preempt the pending communication on $y$, thus resulting in resending the required lock $r$ with state information t. An appropriate notion of barb may therefore be given by observing processes within 'saturating' contexts, but this remains to be investigated.

The same arguments as for the encoding of separate choice with reversed order of testing also hold for the encoding of mixed choice as of Figure 7, because sender-locks are not always the second lock to be tested, as would happen with the above example, when the order determines the sender-lock as smaller than the receiver-lock.

\section{Problem 3: Alien contexts}

Although not important for barbed bisimulation, but rather barbed congruence, let us also comment on the specifics of choice encodings with output-guards in that respect.

Since the internal names $l, a$ become free after an observed output on some high-level channel, we need to require that a context behaves according to the protocol of the encoded 
terms. In general, we cannot expect correct behavior within alien contexts (see also [VP96]), so we could impose the requirement to regard $\Sigma$-contexts only, i.e. only those contexts that can be generated via $\llbracket \rrbracket$-encodings of $\mathbb{S}^{\Sigma}$-contexts, and define: two processes $P$ and $Q$ are called $\Sigma$-barbed congruent, if $C[P] \dot{\bowtie} C[Q]$ for all $\Sigma$-contexts $C[]$.

It would be interesting to investigate, whether some form of typed observation could replace the somewhat delicate notion of $\Sigma$-context (and also of $\Sigma$-barb). Basically, a notion of type should capture, whether some context $C[]$ respects the protocol expected by some process $P$ as if it was a $\Sigma$-context, such that $C[P]$ becomes acceptable. However, the expected protocols for our choice encodings not only require that the pure typing aspects of Kobayashi's reliable channels are respected, but also that the boolean values on the lock channels are correctly handled by some reader of a lock in the context. More precisely, a reader of a lock not only must eventually send back some boolean value - in addition, it must never change the lock's value from $f$ to $t$, but only from $t$ to $f$, or leave it unchanged. This means that value-dependencies, although of a rather simple nature, would have to be included in the 'type' system. Work in that direction is not yet known to the author, but some extension of [Kob97, Yos96] seems worth pursuing.

Summary Altogether, we conclude that the problem of full abstraction for the encoding of separate choice (in comparison to input-guarded choice) might be dealt with in an ad-hoc, although not unfeasible, manner by using tailored $\Sigma$-barbs and -contexts to state some quite specific full abstraction result with respect to " $\Sigma$-barbed coupled $\Sigma$-congruence". The case for mixed choice seems hopeless at first, but, also in this case, we may state something reasonable by carefully choosing a restricted setting, as suggested in the following section.

\subsection{Adequacy via restriction}

In this section, we will manipulate and restrict source and target terms in such a way that all communications involved in the choice protocol will always be internal. This allows us to state quite strong full abstraction properties - although in a restricted setting - since neither gradual commitments nor invalid outputs nor alien contexts come into play.

We explicitly introduce single input- and output-prefixes into the source language $\mathbb{S}^{\text {sep }}$ and we distinguish the channels $\mathbf{N}$ according to their syntactic usage as single names $\mathbf{N}^{\mathbf{s}}$ (appear as subject in single prefixes) or selectable names $\mathbf{N}^{c}$ (appear as subjects in selectable prefixes, i.e. choice-branches). Moreover, we restrict the source language such that communication on selectable channels is always restricted and forbid the passing of selectable channels as objects such that their scope is never extruded; then the only use of selectable channels is as subject of branches in choice expressions and as bound variable in restriction (never in object position of either input or output). These restrictions can be easily imposed by a type system that distinguishes between inner types $\left(I T::=\mathbb{B}|\mathbb{N}|[\tilde{I T}]^{\mathrm{s}}\right)$ and outer types $\left(T::=I T \mid[\tilde{I T}]^{\mathrm{c}}\right)$, where selectable names may only have outer types. For simplicity, we omit replicated inputs on selectable channels. 


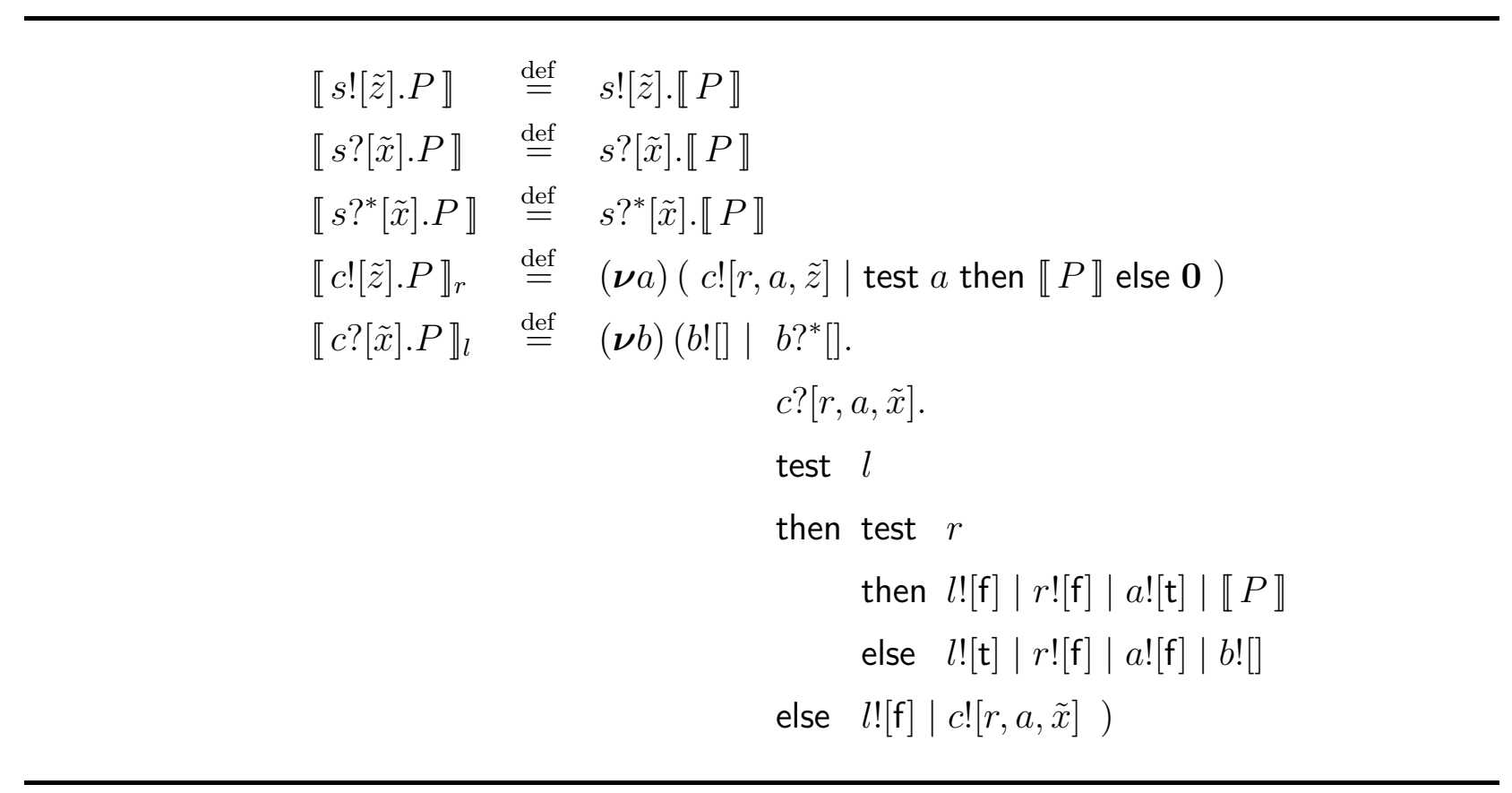

Figure 10: $\mathbb{S}^{\mathrm{sep}} \rightarrow \mathbb{T}^{\prime}$

We choose a target language $\mathbb{T}^{\prime}$ with synchronous output such that output on single channels may be encoded trivially and, thus, it is not possible to observe a broken atomicity by its encoding using asynchronous output. Note that for the same reason that the encodings $\pi_{\mathrm{s}} \rightarrow \pi_{\mathrm{a}}$ (see Figure 1) are not fully abstract with respect to weak bisimulation [Hon92], also the encodings for choice with output-guards in this paper are not: the atomicity of outputs is visibly broken into a send-request and an acknowledgement reception. Consequently, whereas the processes $y ![] . y ![] .0$ and $y ![] .0 \mid y ![] .0$ are weakly bisimilar, their translations are not. In contrast, the broken atomicity of inputs in the encoding of input-guarded choice is not equally visible since the second step is always restricted.

With the above distinctions and restrictions, we rephrase in Figure 10 the definition for the uniform encoding of separate choice as of Figure 5, where we use $s$ to indicate single channels and $c$ to indicate selectable channels. With respect to full abstraction, we shall only be interested in processes that do not exhibit communication on selectable channels to the outside, so we require of $\Sigma$-closed processes $P$ that $f n(P) \cap \mathbf{N}^{c}=\emptyset$, i.e. that no selectable name may occur free in $P$. From the outside, source terms that internally might use selectable channels in choice expressions can then not be distinguished at all from their translations. Formally, within this very restricted setting, we get an indistinguishability result. Let $\lesssim$ denote the expansion preorder [AH92], a refinement of the standard synchronous weak bisimulation that takes the number of internal steps into account, and let us assume a standard labeled operational semantics for this purpose. 
Proposition 5.3.1. For all $\Sigma$-closed $S \in \mathbb{S}^{\text {sep }}: S \lesssim \llbracket S \rrbracket$

Intuitively, this proposition states that 'programming with choice' is implemented correctly.

Proof. (Sketch) There is a tight operational correspondence between transitions in source and target, which can be exploited to exhibit the required expansion relation.

Because of the setup of the encoding, every visible transition is on some name in $\mathbf{N}^{\mathbf{s}}$, and since the encoding leaves them untouched, there is a 1-1 correspondence, here.

For internal transitions on names in $\mathbf{N}^{\mathbf{s}}$, there is again a $1-1$ correspondence. The only interesting case is for reductions on names in $\mathbf{N}^{c}$. Here, one high-level step corresponds to a sequence of (at least 4) reductions resulting in the desired state plus some active garbage processes that represent unchosen branches. Due to $\Sigma$-closedness, these garbage processes do just potentially generate additional internal reductions without ever changing the visible behavior, so a term $\llbracket S \rrbracket \Rightarrow T$ after having committed by performing the two tests is actually just an expansion of the high-level descendant $S \rightarrow S^{\prime}$ with $T \gtrsim \llbracket S^{\prime} \rrbracket$.

The required relation (actually an 'expansion up to expansion') can then be constructed by pairing all $S \in \mathbb{S}^{\text {sep' }}$ with the bisimilar descendants $T$ of $\llbracket S \rrbracket$, i.e., with $\llbracket S \rrbracket \Rightarrow T$ and $\llbracket S \rrbracket \approx T$. Note that this proof carries through not only for output transitions, but also for input transitions, the result holds for 'synchronous' expansion.

From Proposition 5.3.1, we get full abstraction for free.

Corollary 5.3.2. for all $S_{1}, S_{2} \in \mathbb{S}^{\mathrm{sep}}: S_{1} \approx_{s} S_{2}$ iff $\llbracket S_{1} \rrbracket \approx_{s} \llbracket S_{2} \rrbracket$

Proof. By the fact that expansion refines weak bisimulation, and by transitivity.

Completely analogous, restricted full abstraction also results hold for the other choice encodings of Figures 6, 7, and 8, when considered in the restricted setting with single and selectable channels and observed only under the regime of $\Sigma$-closedness; however, the possibility for divergence is not taken into account by expansion relations.

Another result that we get for free from the direct comparison of source terms and their translations in Proposition 5.3.1, is about deadlock-freedom.

Corollary 5.3.3. The encoding $\llbracket \rrbracket: \mathbb{S}^{\mathrm{sep} \prime} \rightarrow \mathbb{T}^{\prime}$ is deadlock-free.

Proof. The fact that expansion refines weak bisimulation tell us that any computation path chosen starting from some translation $\llbracket S \rrbracket$ is tied to some high-level computation path, and if this high-level path has further possible steps, the low-level can always mimic it. 


\section{Conclusion}

The encodings presented in this paper should exhibit how to abstractly model distributed implementations of guarded choice within the asynchronous $\pi$-calculus. Prompted by Palamidessi's work [Pal97], we emphasized the problematic case of mixed choice by developing first the quite simpler encoding for separate choice. Whereas this case satisfies all of Palamidessi's required properties, the transition to encodings for mixed choice bears all of the awkwardnesses. Two sources of potential deadlock are identified: cyclic waiting on lock channels and 'incestuous' self-communication. In order to cope with them, we pointed out that either uniformity or divergence-freedom must be dropped, if we want to stay within the chosen framework, thus confirming Palamidessi's negative result. Furthermore, we motivated that slight changes to the framework allow us to overcome the impossibility, and we exposed full abstraction results that can be achieved for the various choice encodings.

Since our encodings of mixed choice and the proposed variants in $\S 4$ can be seen as abstractions of practically 'good' distributed implementations [Kna93], one interpretation of our work might be an evaluation of whether Palamidessi's criteria are too strong for practical purposes. It was pointed out quite early [RL94] that probabilistic solutions - with divergence, but without livelock and with progress probability 1-might be practical, although they are not reasonable in [Pal97]. On the other hand, the standard way of implementing channel managers as autonomous threads [Kna93, LT95] contradicts the requirement of uniformity, if open systems are considered. As our work shows, relaxing uniformity by admitting a toplevel context or relaxing reasonableness to admit some fair degree of well-behaved divergence renders many practically motivated encodings theoretically 'good'.

In the spirit of Bougé's informal notion of symmetry "there is no priority or any other form of externally specified static partial ordering among processes" [Bou88], we may note that none of the branches in choices is statically assigned priority over its competitors by the encoding in Figure 7-the symmetry is broken only dynamically by taking a totally-ordered ticket when entering the 'bakery'. In accordance with Rabin and Lehmann [RL94], we needed (only) one small piece of global memory: the protected message on channel $c$.

Concerning the 'bakery primitive' that we introduced for the $\pi_{\mathrm{a}}^{\kappa}$-calculus, we should add that it is, of course, problematic to give a standard reduction semantics for this primitive. Processes would need to store the identification numbers with them and be able to access them when required to compare them. A variant of 'configurations' containing a process and a set of identifiers, as used in some semantics of Facile [TLK96], might be used here. Yet, as we mentioned earlier, a calculus with such a primitive should be considered as instrinsically asymmetric since it allows the programmer to dynamically generate asymmetries, like timestamps, among processes and to use these for decisions when needed. We believe such calculi deserve further study.

Finally, it is necessary to point out that all of the work reported within this paper does not at all strive to tackle the problems of space leaks and efficiency, which naturally need to be considered for practical implementations. Actually, even for the encoding of input-guarded 
choice, there are examples demonstrating that garbage collection of unused branches is not not always possible and may lead to unwantedly growing terms.

\section{Acknowledgements}

The author would like to thank Cédric Fournet, Kohei Honda, Naoki Kobayashi, Catuscia Palamidessi, Benjamin Pierce, Davide Sangiorgi, Martin Steffen, and Nobuko Yoshida for many fruitful discussions, and the anonymous referees for their constructive remarks.

\section{A Channel managers are not uniform}

Distributed implementations of channel-based communication usually employ so-called channel manager processes CHAN $(y)$ for mediating between the activities of senders and receivers on channel $y$. Often, this is done by collecting send- and receive-requests in queues that are attached to channel managers; the synchronization protocol for a particular channel is then only started when a pair of complementary requests (from different choices) can be found in the respective queue [Kna93, LT95]. ${ }^{6}$

In $\pi$-calculus encodings, the creation of channel managers would have to take place at the moment the corresponding channel name is created:

$$
\llbracket(\boldsymbol{\nu} y) P \rrbracket \stackrel{\text { def }}{=}(\boldsymbol{\nu} y)(\llbracket P \rrbracket \mid \operatorname{CHAN}(y))
$$

However, free names in process terms would, in their encoding, have to be supplied explicitly with their managers at the top-level of encodings:

$$
\llbracket P \rrbracket \stackrel{\text { def }}{=} \llbracket P \rrbracket \mid \prod_{x \in f n(P)} \operatorname{CHAN}(x)
$$

This, in turn, conflicts with Palamidessi's requirement of uniformity, since the equation $\llbracket P \rrbracket|\llbracket Q \rrbracket=\llbracket P| Q \rrbracket$ does not hold in general, because a free name shared by $P$ and $Q$ would be provided with two competing managers on the left side, but only one (as intended) on the right side. For this reason, e.g. encodings with 'centralized' channel managers are not uniform. Consequently, if we want to stick to 'uniformity', we either have to restrict ourselves to closed process terms with no observable behavior at all (i.e. no free names), or we have to leave the encoding of restriction as

$$
\llbracket(\boldsymbol{\nu} y) P \rrbracket \stackrel{\text { def }}{=}(\boldsymbol{\nu} y) \llbracket P \rrbracket
$$

and distribute the functionality of channel managers, if possible, over all places where channels are used, as is exemplified in the encodings of $\S 3$ and $\S 4$.

\footnotetext{
${ }^{6}$ In a distributed system, channel managers need to reside at some particular location, the choice of which may heavily influence the efficiency of computations: it decides whether individual communications are either local or remote. Consequently, a big advantage is the unique-receiver property (see $\S 4.3$ and [FG96]), because it allows the implementer to statically choose just the location of the unique receiver as the residence of the channel manager process.
} 


\section{B Type-checking partial deadlock-freedom}

This section provides a quick overview of Kobayashi's type system for deadlock-freedom. Since it would be too space-consuming to present all the necessary formal definitions and theorems from [Kob97], we assume the more interested reader to have a copy of that paper at hand. We then provide the ingredients for carrying out the formal proof for an application of this type system to the encoding of separate choice as in Figure 5.

\section{B.1 Types for reliable channels}

We sketch Kobayashi's [Kob97] non-recursive channel types $p^{m}\left[T_{1}, \ldots, T_{n}\right]^{t}$ with

- polarity $p \subseteq\{I, O\}$ known from Pierce and Sangiorgi [PS96] for denoting input and output capabilities (with abbreviations $\mid:=\{\}, \uparrow:=\{O\}, \downarrow:=\{I\}$, and $\uparrow:=\{I, O\}$ ),

- multiplicity $m \in\{1, *, M, \omega\}$ for classifying channels,

- arity $n \in \mathbb{N}_{0}$ known from Milner's polyadic $\pi$-calculus [Mil93], and

- time tag $t \in \mathbf{T}_{\star}$, which we explain below.

The multiplicity of a channel constrains its usage according to capabilities and obligations: linear (1) channels must eventually be used once for input and output, after they become active; replicated-input $(*)$ channels must be used in exactly one replicated input-prefix immediately after creation, but they can be used arbitrarily often for sending; mutex $(M)$ channels have to be supplied with some value immediately after creation, and a reader of some mutex channel is obliged to eventually resend some value, thus, at any time, there is at most one message in the system; unreliable $(\omega)$ channels can be used arbitrarily with the exception of replicated input-prefixes. A channel is also called reliable if it is not unreliable; basically, every process that communicates on a reliable channel will eventually find its communication partner.

Kobayashi introduces a type system for a calculus very similar to our target language $\mathbb{T}$ with boolean primitives ( $\mathbb{B}$ as base type, and if instead of test) and a typed restriction operator, which also introduces fresh time tags. (For some proof strategies, it also convenient to introduce additional time tags with input variables, which then have to match the tags of the communicated values.) Time tags $t$ are used within the typing rules to express constraints on the order of using reliable channels (unreliable channels as well as replicated input channels always carry the tag $\star$ which is both smaller and greater than any other tag): basically, $s \prec t$ means that obligations according to the type of a channel associated with $t$ may (not must) be delayed until the completion of some communication on the channel associated with s. Example obligations are, for example, that a linear channel needs to be find the complementary partner, once it has become active; with mutex channels, only the sender half represents an obligation: if the channel associated with tag $t$ is a mutex channel, then the (re-) sending on channel may be blocked by some communication on the channel associated with tag $s$. Clearly, deadlocks may arise when the ordering among the channels indicates cyclic waiting, 
where an obligation might, by transitivity, be blocked by its own fulfillment. Thus, an ordering $\prec$ is indicating deadlock-freedom, if its transitive closure $\prec^{+}$is a strict partial order on $\mathbf{T} \times \mathbf{T}$ (i.e. ignoring the $\star$ ).

Kobayashi then specifies partial deadlock-freedom of terms by means of his classification of channels: only the reliable channels are expected to be satisfy deadlock-freedom properties. For explanation, let us assume a process $P \nrightarrow$ with no more reductions. Let us further regard $P$ in normal form, i.e. $P \equiv(\boldsymbol{\nu} \tilde{w}) \prod N_{j}$ where $N$ are either a message $y ![\tilde{z}]$, a single input $y ?[\tilde{x}] . Q$, or a replicated input $y ?^{*}[\tilde{x}] . Q$. Whether $N$ is interpreted as a deadlocked sub-process in $P$, depends on the type of its channel $y$ :

- According to the intuition of replicated and mutex channels, mutex messages and replicated receivers are not counted as regards deadlock, because they are meant to be always (for mutex: always eventually) present after channel creation. On the other hand, mutex receivers and replication triggers indicate unwanted deadlock, since they are meant to always find their counterpart, so $N$ should be neither of them.

- With respect to linear channels, $N$ must be seen in context. If its channel $y$ is restricted in $P$, then we regard $N$ as causing deadlock, since it was never used after it has become active; if this $y$ is free in $P$, then its complement might still be supplied from the outside, and then $y$ is called half-used.

- If $N$ 's channel $y$ is unreliable, then it is not interpreted as ill-behaved since we have not required it to behave reliably in any sense.

Let $\Gamma$ be a list of typing assumptions for names, and $\prec$ a tag ordering, where $\prec^{+}$is a strict partial order. A typing judgement $\Gamma, \prec \vdash P$ intuitively means

1. $P$ uses only the capabilities specified in $\Gamma$,

2. $P$ fulfills all the obligations specified by $\Gamma$, and

3. $P$ obeys the ordering specified in $\prec$ in fulfilling the obligations.

There are two strategies for using Kobayashi's type system:

(A) One uses Kobayashi's two-phase type-checking algorithm: the first-phase is building up a type derivation tree witnessing the correct use of arities, polarities, and multiplicities, while collecting up constraints on time tags according to the applied rules; the second phase tries to solve the tag constraints and checks whether the transitive closure of the resulting tag ordering, if it exists, is a strict partial order.

(B) One supplies an appropriate candidate for a time tag ordering from the beginning and directly type-checks the terms under investigation in one single phase including the conditions on time tag required by the applied rules. 
For well-typed processes, we have the following useful properties concerning partial deadlockfreedom [Kob97], which we present here in a weaker, but simplified version:

\section{Theorem B.1.1 (Subject reduction).}

If $\Gamma, \prec \vdash P$ and $P \rightarrow P^{\prime}$, then there are $\Gamma^{\prime}, \prec^{\prime}$ with $\Gamma^{\prime}, \prec^{\prime} \vdash P^{\prime}$.

Theorem B.1.2 (Immediate deadlock). Suppose $\Gamma, \prec \vdash P$ and $P \nrightarrow$, then pending communications in $P$ are (1) on some unreliable channel, (2) on some linear channel that is half-used according to $\Gamma$, or (3) are either a mutex message or a replicated input.

Together, the two theorems guarantee that during reduction of well-typed processes no partial deadlock, as specified by the classification of reliable channels, can ever occur.

\section{B.2 Separate choice}

In this subsection, we prove deadlock-freedom for the encoding of separate choice in Figure 5 via Kobayashi's type system in the following sense: since we use only reliable channels for encoding choice, and if we regard all high-level channels as unreliable, then the typability of translated terms implies that the encoding does not add deadlocks. This is true, because all of these reliable channels - especially the linear acknowledgement channels - are restricted, so only the cases (1) and (3) of Theorem B.1.2 apply. Since mutex messages and replicated inputs of case (3) can be regarded as garbage, the only remaining deadlocked subprocesses are on high-level channels. Since these have been translated in a $1-1$ fashion, we know that the deadlock must have been present already in the source term.

According to the above idea, we first assume a simple polyadic type system for the source language as a non-recursive structural variant of Milner [Mil93], extended with boolean-typed names. This system can be seen as Kobayashi's system by stripping off polarity, multiplicity, and time tags, or else by having only one polarity $\uparrow$, one multiplicity $\omega$, and one time tag $\star$, such that $[T]:=\downarrow^{\omega}[T]^{\star}$, where $T$ is either the type boolean $\mathbb{B}$ or a finite tuple $\tilde{T}$. Let us assume that we have typing statements of the form $\{\tilde{y}: \tilde{T}\}=: \mathcal{T} \vdash P$ in $\mathbb{S}$, and that all source terms under investigation are well-typed, accordingly.

Next, we propose a tag ordering that we are going to apply for type-checking translated terms (i.e. we are following proof strategy B). It is intuitively derived from the encoding's algorithmic idea. Let $\mathbf{T} \supseteq \mathbf{T}_{l} \uplus \mathbf{T}_{r} \uplus \mathbf{T}_{a}$ distinguish three pairwise disjoint subsets of tags, where the indices of the tag sets indicate the channels they are going to be associated with.

$$
\prec \stackrel{\text { def }}{=}\left(\mathbf{T}_{l} \times \mathbf{T}_{a}\right) \cup\left(\mathbf{T}_{r} \times \mathbf{T}_{a}\right) \cup\left(\mathbf{T}_{r} \times \mathbf{T}_{l}\right)
$$

defines the tag ordering that corresponds to the use of mutex and linear channels in the encoding: a communication on the linear acknowledgement channels $a$ may be delayed by both a communication a receiver-lock $l\left(\mathbf{T}_{l} \times \mathbf{T}_{a}\right)$ or on a sender-lock $r\left(\mathbf{T}_{r} \times \mathbf{T}_{a}\right)$, whereas the resending of the mutex message for the receiver-lock $l$ may be blocked by a reception for the 


$$
\begin{aligned}
& l: \uparrow^{M}\left[\mathbb{B}^{\star}\right]^{t_{l}} \quad r: \uparrow^{M}\left[\mathbb{B}^{\star}\right]^{t_{r}} \quad a: \uparrow^{1}\left[\mathbb{B}^{\star}\right]^{t_{a}} \quad b: \uparrow^{*}[]^{\star} \\
& y: \llbracket[\tilde{T}] \rrbracket^{\mathbf{T}} \stackrel{\text { def }}{=} \uparrow^{\omega}\left[\downarrow^{M}\left[\mathbb{B}^{\star}\right]^{t_{r}}, \uparrow^{1}\left[\mathbb{B}^{\star}\right]^{t_{a}}, \llbracket \tilde{T} \rrbracket^{\mathbf{T}}\right]^{\star} \quad \text { in } \mathbb{T}, \quad \text { if } y:[\tilde{T}] \text { in } \mathbb{S}
\end{aligned}
$$

Figure 11: Types for $\mathbb{S}^{\text {sep }} \rightarrow \mathbb{T}$

sender-lock $r\left(\mathbf{T}_{r} \times \mathbf{T}_{l}\right)$, but not the other way around. The latter blocking actually happens only if the receiver-lock carried t, so only then it will not be resent until the sender-lock has also been checked. The following important fact holds by definition:

Lemma B.2.1. $\prec^{+}$is a strict partial order.

With this ordering, we can now provide a typed encoding $\llbracket \rrbracket^{\mathbf{T}}$ for separate choice, assuming that we only consider well-typed source terms. In Figure 11, each of the channels that are added by the encoding is given a reliable type and is associated the respective time tag, where $t_{l} \in \mathbf{T}_{l}, t_{r} \in \mathbf{T}_{r}$, and $t_{a} \in \mathbf{T}_{a}$. In contrast, every high-level channel is regarded as unreliable (note $y$ 's multiplicity $\omega$ ), and its type is appropriately translated to carry additional information according to its use in encoded terms: a low-level send-request on a high-level channel $y$ carries the input-end for some mutex channel $\left(\downarrow^{M}\left[\mathbb{B}^{\star}\right]^{t_{r}}\right)$ and the output-end for some linear acknowledgement channel $\left(\uparrow^{1}\left[\mathbb{B}^{\star}\right]^{t_{a}}\right)$ as additional parameters. With $\llbracket \mathbb{B} \rrbracket^{\mathbf{T}}:=\mathbb{B}^{\star}$, let us also extend the typed encoding on types componentwise to type environments $\mathcal{T}$ of the source language by $\llbracket \emptyset \rrbracket^{\mathbf{T}}:=\emptyset$ and $\llbracket \mathcal{T}, y: T \rrbracket^{\mathbf{T}}:=\llbracket \mathcal{T} \rrbracket^{\mathbf{T}}, y: \llbracket T \rrbracket^{\mathbf{T}}$.

On terms, the typed encoding $\llbracket \rrbracket^{\mathbf{T}}$ is then defined by simply adding the types proposed in Figure 11 for $a, b, l$ and $r$, to the restriction occurrences in Figure 5, where each occurrence gets a fresh time tag. We further translate the typed restriction $(\boldsymbol{\nu} x: T) P$ for high-level channels in the source language into the enhanced typed restriction $\left(\boldsymbol{\nu} x: \llbracket T \rrbracket^{\mathbf{T}}\right) \llbracket P \rrbracket^{\mathbf{T}}$ in the target language. For type-checking, we expand out test-expressions by using if-forms instead, and we also add time-tags in the case of the encoding of (replicated) inputs, so let us choose occurrences of tag variables $s_{r} \in \mathbf{T}_{r}$ and $s_{a} \in \mathbf{T}_{a}$ and attach them to the respective occurrences in the encoding of Figure 5. The third parameter of translations of high-level inputs $\tilde{x}$ is, according to Figure 11, implicitly tagged with $\star$.

Lemma B.2.2. $\left(\left\{\left[t_{r}, t_{a}\right] /\left[s_{r}, s_{a}\right]\right\}\right) \prec \subseteq \prec$

The order of the time tags $t$ of the received channels $r$ and $a$ is always sufficient for the required order on the time tags $s$ of the corresponding input variables. This lemma is needed, whenever we derive low-level communications on high-level channels $y$.

Lemma B.2.3. $\left\{t_{r}, t_{a}\right\} \nprec \nsucc\left\{s_{r}, s_{a}\right\}$

Note that $t_{r} \prec s_{l} \prec s_{a}$ (and $s_{r} \prec s_{l} \prec t_{a}$ ), but that $t_{r} \nprec s_{a}$ (and $s_{r} \nprec t_{a}$ ), since $\prec$ is not transitive. This lemma is needed in the proof of IN-rules. 
Since we interpret all high-level channels as unreliable, we face the problem, for the type-checking of typed translations, that rule T-IN of Kobayashi [Kob97] is not applicable, when trying to use it for a high-level replicated input. So, we need to assume an additional typing rule T-URIN (unreliable replicated input), which is defined just like T-IN of Kobayashi [Kob97], but allows the conclusion for replicated input syntactically. This rule does not change the deadlock-properties of the well-typed processes, if we only allow its application for channels that we explicitly regard as unreliable. Finally, we get the main property, where $\prec \downarrow_{\mathbf{I}\left(\llbracket S \rrbracket^{\mathbf{T}}\right)}$ denotes $\prec$ with all ordered pairs mentioning inner tags $\mathbf{I}\left(\llbracket S \rrbracket^{\mathbf{T}}\right)$ of the translated term, i.e. those introduced as $s_{r}$ and $s_{a}$ above, removed.

Proposition B.2.4 (Preservation of typing).

Let $S \in \mathbb{S}^{\text {sep }}$. If $\mathcal{T} \vdash S$, then $\left(\llbracket \mathcal{T} \rrbracket^{\mathbf{T}} \cup\left\{\mathrm{t}, \mathrm{f}: \mathbb{B}^{\star}\right\}\right), \prec \downarrow_{\mathbf{I}\left(\llbracket S \rrbracket^{\mathbf{T}}\right)} \vdash \llbracket S \rrbracket^{\mathbf{T}}$.

Proof. By induction on the structure of $S$.

The intuition of well-typed processes is that deadlocks can only be caused by unreliable channels, so we also know that deadlocks can only be caused by high-level channels: every deadlock in some derivative originates from some deadlock present in the source language.

Corollary B.2.5. $\mathbb{S}^{\mathrm{sep}} \rightarrow \mathbb{T}$ is deadlock-free.

\section{B.3 Mixed choice}

In analogy to the discussion on cyclic waiting ( $\S 4$ ), the type-checker (strategy A) for deadlockfreedom fails, when reusing the encoding for separate choice in the case of mixed choice. In type-checking the example $\llbracket P \mid Q \rrbracket$, there is no ordering for the use of the two choice-locks that can be used consistently on both sides of the parallel composition (cf. proof sketch of Proposition B.2.4). Although Kobayashi's system is not complete, i.e. it rejects processes that are deadlock-free, in our case the rejection is correct as indicated in $\S 3$.

Similar to the approach in the previous section, we can provide a tag ordering for the randomized solution of Subsection 4.1: the pairs are given by $t_{s} \prec t_{b} \prec t_{l}, t_{r}, t_{a}$, where we again indicate the ordering among (sets of) tags by their associated channel names. The state channels $s$ associated with tags $t_{s}$ are never delayed since resending on $s$ is always immediate, according to the encoding in Figure 6 . With Kobayashi, we may say that well-typed terms never get into a deadlock, however they might fall into an infinite loop.

If we wanted to use Kobayashi's system to type-check the 'bakery' encoding for mixed choice in Figure 7, we would need to extend the system to deal with natural numbers. Then, we would need rules for checking consistency of the constraints on the time tag ordering on critical channels with the occurrences of the conditional operator: in contrast to Kobayashi's rules, we would need to allow the two strands of a conditional to be typed according two different time tag orderings. The study of the feasibility of such a system is left for future work. 


\section{References}

[ACS98] R. M. Amadio, I. Castellani and D. Sangiorgi. On Bisimulations for the Asynchronous $\pi$-Calculus. Theoretical Computer Science, 195(2):291-324, 1998. An extended abstract appeared in Proceedings of CONCUR '96, LNCS 1119: 147-162.

[AH92] S. Arun-Kumar and M. Hennessy. An Efficiency Preorder for Processes. Acta Informatica, 29:737$760,1992$.

[Ama97] R. M. Amadio. An Asynchronous Model of Locality, Failure, and Process Mobility. In D. Garlan and D. Le Metayer, eds, Proceedings of COORDINATION '97, volume 1282 of LNCS. Springer, 1997. Extended version as Rapport de Recherche RR-3109, INRIA Sophia-Antipolis, 1997.

[Ben83] M. Ben-Or. Another Advantage of Free Choice: Completely Asynchronous Agreement Protocols (Extended Abstract). In Proceedings of PODC '83, pages 27-30. ACM, August 1983.

[Ber80] A. Bernstein. Output Guards and Nondeterminism in "Communicating Sequential Processes". ACM Transactions on Programming Languages and Systems, 2(2):234-238, Apr. 1980.

[BGZ97] N. Busi, R. Gorrieri and G. Zavattaro. On The Turing-Equivalence of Linda Coordination Primitives. In C. Palamidessi and J. Parrow, eds, Proceedings of EXPRESS '97, volume 7 of ENTCS. Elsevier Science Publishers, 1997.

[Bou88] L. Bougé. On the Existence of Symmetric Algorithms to Find Leaders in Networks of Communicating Sequential Processes. Acta Informatica, 25(2):179-201, 1988.

[Bou92] G. Boudol. Asynchrony and the $\pi$-calculus (Note). Rapport de Recherche 1702, INRIA SophiaAntipolis, May 1992.

[BS83] G. Buckley and A. Silberschatz. An Effective Implementation for the Generalized Input-Output Construct of CSP. ACM Transactions on Programming Languages and Systems, 5(2):223-235, 1983.

[BS94] G. Bellin and P. Scott. On the $\pi$-Calculus and Linear Logic. Theoretical Computer Science, 135:11-65, 1994. Also published as LFCS report ECS-LFCS-92-232, LFCS, University of Edinburgh.

[FG96] C. Fournet and G. Gonthier. The Reflexive Chemical Abstract Machine and the Join-Calculus. In J. G. Steele, ed, Proceedings of POPL '96, pages 372-385. ACM, Jan. 1996.

[FG98] C. Fournet and G. Gonthier. A Hierarchy of Equivalences for Asynchronous Calculi. In K. G. Larsen, S. Skyum and G. Winskel, eds, Proceedings of ICALP '98, volume 1443 of LNCS, pages 844-855. Springer, July 1998.

[Hon92] K. Honda. Notes on Soundness of a Mapping from $\pi$-calculus to $\nu$-calculus. With comments added in October 1993, May 1992.

[HT92] K. Honda and M. Tokoro. On Asynchronous Communication Semantics. In M. Tokoro, O. Nierstrasz and P. Wegner, eds, Object-Based Concurrent Computing 1991, volume 612 of LNCS, pages 21-51. Springer, 1992.

[Kna93] F. Knabe. A Distributed Protocol for Channel-Based Communication with Choice. Computers and Artificial Intelligence, 12(5):475-490, 1993.

[Kob97] N. Kobayashi. A Partially Deadlock-Free Typed Process Calculus. In Proceedings of LICS '97, pages 128-139. Computer Society Press, July 1997. Full version as as Technical Report 97-02, University of Tokyo.

[KS97] D. Kumar and A. Silberschatz. A Counter-Example to an Algorithm for the Generalized InputOutput Construct of CSP. Information Processing Letters, 61:287, 1997. 
[Lam74] L. Lamport. A New Solution of Dijkstra's Concurrent Programming Problem. Journal of the ACM, 17(8):453-455, 1974.

[LT95] L. Leth and B. Thomsen. Some Facile Chemistry. Formal Aspects of Computing, 7(3):314-328, 1995. A Previous Version appeared as ECRC-Report ECRC-92-14.

[Mil93] R. Milner. The Polyadic $\pi$-Calculus: A Tutorial. In F. L. Bauer, W. Brauer and H. Schwichtenberg, eds, Logic and Algebra of Specification, volume 94 of Series F: Computer and System Sciences. NATO Advanced Study Institute, Springer, 1993. Available as Technical Report ECS-LFCS-91-180, University of Edinburgh, October 1991.

[MPW92] R. Milner, J. Parrow and D. Walker. A Calculus of Mobile Processes, Part I/II. Information and Computation, 100:1-77, Sept. 1992.

[MS92] R. Milner and D. Sangiorgi. Barbed Bisimulation. In W. Kuich, ed, Proceedings of ICALP '92, volume 623 of $L N C S$, pages 685-695. Springer, 1992.

[Nes96] U. Nestmann. On Determinacy and Nondeterminacy in Concurrent Programming. PhD thesis, Technische Fakultät, Universität Erlangen, November 1996. Arbeitsbericht IMMD-29(14).

[NP96] U. Nestmann and B. C. Pierce. Decoding Choice Encodings. In U. Montanari and V. Sassone, eds, Proceedings of CONCUR '96, volume 1119 of LNCS, pages 179-194. Springer, 1996. Latest full version as report BRICS-RS-99-42, Universities of Aalborg and Århus, Denmark, 1999. To appear in Journal of Information and Computation.

[Pal97] C. Palamidessi. Comparing the Expressive Power of the Synchronous and the Asynchronous $\pi$ calculus. In Proceedings of POPL '97, pages 256-265. ACM, Jan. 1997.

[PS92] J. Parrow and P. Sjödin. Multiway Synchronization Verified with Coupled Simulation. In R. Cleaveland, ed, Proceedings of CONCUR '92, volume 630 of LNCS, pages 518-533. Springer, 1992.

[PS96] B. C. Pierce and D. Sangiorgi. Typing and Subtyping for Mobile Processes. Mathematical Structures in Computer Science, 6(5):409-454, 1996. An extract appeared in Proceedings of LICS '93: 376-385.

[Rep91] J. Reppy. CML: A Higher-Order Concurrent Language. In Proceedings of PLDI '91, pages 293-259. ACM, June 1991. In SIGPLAN Notices 26(6).

[RL94] M. O. Rabin and D. Lehmann. On the Advantages of Free Choice: A Symmetric and Fully Distributed Solution to the Dining Philosophers Problem. In A. W. Roscoe, ed, A Classical Mind: Essays in Honour of C.A.R. Hoare, chapter 20, pages 333-352. Prentice Hall, 1994. An extended abstract appeared in Proceedings of POPL'81, pages 133-138.

[San93] D. Sangiorgi. Expressing Mobility in Process Algebras: First-Order and Higher-Order Paradigms. $\mathrm{PhD}$ thesis, LFCS, University of Edinburgh, 1993.

[San97] D. Sangiorgi. The Name Discipline of Uniform Receptiveness. In P. Degano, R. Gorrieri and A. Marchetti-Spaccamela, eds, Proceedings of ICALP '97, volume 1256 of LNCS, pages 303-313. Springer, 1997. Full version as Technical Report, INRIA Sophia-Antipolis, December 1996.

[TLK96] B. Thomsen, L. Leth and T.-M. Kuo. A Facile Tutorial. In U. Montanari and V. Sassone, eds, Proceedings of CONCUR '96, volume 1119 of LNCS, pages 278-298. Springer, 1996.

[VP96] B. Victor and J. Parrow. Constraints as Processes. In U. Montanari and V. Sassone, eds, Proceedings of CONCUR '96, volume 1119 of LNCS, pages 389-405. Springer, 1996.

[Yos96] N. Yoshida. Graph Types for Monadic Mobile Processes. In V. Chandru and V. Vinay, eds, Proceedings of FSTTCS '96, volume 1180 of LNCS, pages 371-386. Springer, 1996. Full version as Technical Report ECS-LFCS-96-350, University of Edinburgh. 


\section{Recent BRICS Report Series Publications}

RS-99-43 Uwe Nestmann. What is a 'Good' Encoding of Guarded Choice? December 1999. ii+34 pp. To appear in a special EXPRESS '97 issue of Information and Computation. This revised report supersedes the earlier BRICS report RS-97-45.

RS-99-42 Uwe Nestmann and Benjamin C. Pierce. Decoding Choice Encodings. December 1999 . ii+62 pp. To appear in Journal of Information and Computation. An extended abstract appeared in Montanari and Sassone, editors, Concurrency Theory: 7th International Conference, CONCUR '96 Proceedings, LNCS 1119, 1996, pages 179-194.

RS-99-41 Nicky O. Bodentien, Jacob Vestergaard, Jakob Friis, Kåre J. Kristoffersen, and Kim G. Larsen. Verification of State/Event Systems by Quotienting. December 1999. 17 pp. Presented at Nordic Workshop in Programming Theory, Uppsala, Sweden, October 6-8, 1999.

RS-99-40 Bernd Grobauer and Zhe Yang. The Second Futamura Projection for Type-Directed Partial Evaluation. November 1999. Extended version of an article to appear in Lawall, editor, ACM SIGPLAN Workshop on Partial Evaluation and SemanticsBased Program Manipulation, PEPM '00 Proceedings, 2000.

RS-99-39 Romeo Rizzi. On the Steiner Tree $\frac{3}{2}$-Approximation for QuasiBipartite Graphs. November 1999. 6 pp.

RS-99-38 Romeo Rizzi. Linear Time Recognition of $P_{4}$-Indifferent Graphs. November 1999. 11 pp.

RS-99-37 Tibor Jordán. Constrained Edge-Splitting Problems. November 1999. 23 pp. A preliminary version with the title Edge-Splitting Problems with Demands appeared in Cornujols, Burkard and Wöginger, editors, Integer Programming and Combinatorial Optimization: 7th International Conference, IPCO '99 Proceedings, LNCS 1610, 1999, pages 273-288.

RS-99-36 Gian Luca Cattani and Glynn Winskel. Presheaf Models for CCS-like Languages. November 1999. ii+46 pp.

RS-99-35 Tibor Jordán and Zoltán Szigeti. Detachments Preserving Local Edge-Connectivity of Graphs. November 1999. 16 pp. 